\title{
A Study of the Impact of Polymer-Surfactant in Drug Nanoparticle Coated Pharmatose Composites on Dissolution Performance
}

\author{
Meng Li, Nicole Lopez, Ecevit Bilgili* \\ Otto H. York Department of Chemical, Biological and Pharmaceutical Engineering, \\ New Jersey Institute of Technology, University Heights, Newark, NJ 07012, USA
}

Keywords: Drug nanoparticles, Stabilization, Wet media milling, Fluidized bed coating, Fast dissolution

*Corresponding author: Ecevit Bilgili, New Jersey Institute of Technology. Otto H. York Department of Chemical, Biological, and Pharmaceutical Engineering, 161 Warren St. 150 Tiernan Hall, Newark, NJ 07102, USA. E-mail: bilgece@njit.edu, Tel.: +1 973596 2998; Fax: +1 9735968436.

(C) 2016. This manuscript version is made available under the Elsevier user license http://www.elsevier.com/open-access/userlicense/1.0/ 


\begin{abstract}
Without a proper stabilizer formulation, drug nanoparticles can aggregate during the formation of nanosuspensions and their drying into composite powders, which in turn can cause inadequate bioavailability enhancement from nanoparticles and ensuing lack of therapeutic efficacy. In this study, formulations with various polymer concentrations-molecular weights in the presence/absence of a surfactant were explored to assess their impact on redispersibility and drug dissolution from the composites. Suspensions of griseofulvin (GF), a poorly water-soluble drug, were prepared by wet stirred media milling and subsequently dried via fluidized bed dryingcoating onto Pharmatose ${ }^{\circledR}$ carrier particles. Hydroxypropyl cellulose (HPC), with various molecular weights, sodium dodecyl sulfate (SDS), and their combinations were used as stabilizers during the milling. The dried composites were redispersed in water and SDS solution to recover the GF nanoparticles. Particle sizing via laser diffraction, SEM imaging, and dissolution testing were performed to investigate the redispersion and dissolution performance. Results show that good physical stability of the milled suspension is necessary, but not sufficient to guarantee fast redispersion-drug dissolution. For best performance, presence of SDS is critical; a minimum concentration of the polymer, as a film former, is also required to prevent the formation of hard aggregates during drying.
\end{abstract}




\section{Introduction}

Bioavailability of a large percentage of newly discovered drug compounds is limited by their poor water solubility and slow dissolution rate [1]. Production of drug nanoparticles, typically particles smaller than $1 \mu \mathrm{m}$ in prevalent pharmaceutical literature, has been commonly used to enhance the bioavailability of such drugs. Size reduction of drug crystals increases the specific surface area, which can improve the dissolution rate of drugs $[2,3]$, according to the NoyesWhitney equation [4]. Drug nanoparticles can be produced by "bottom-up" or "top-down" technologies, or combinations of the two [5]. The bottom-up approach refers to methods that create small drug particles from drug molecules dissolved in an organic solution [6] such as in liquid anti-solvent precipitation, precipitation using supercritical fluid, and evaporative precipitation. Top-down approach includes wet media milling [7] and high pressure homogenization [8]. Wet media milling has been the most popular approach to prepare drug nanosuspensions, as it has the distinct advantages of high drug loading and low excipient side effects, and it can be generally applicable to most drug candidates [5].

To meet high patient/clinical demand for solid dosage forms, nanosuspensions are usually converted via drying into nanocomposite microparticles, which will be referred as the drug composites throughout the paper, and incorporated into standard solid dosage forms such as capsules, tablets, and sachets [9-11]. Drying of nanosuspensions can be achieved via spray drying [12, 13], spray-freeze drying [14, 15], freeze drying [16, 17], vacuum drying [18, 19], as well as granulation with, or coating onto, inert excipient particles $[9,20]$. Fluidized bed drying of the drug nanosuspensions can be used to produce layered drug composites [21], which are very attractive because the fluidized bed technology has already been used in pharmaceutical industry widely for drying, granulating, and coating. 
Unfortunately, drug nanoparticles tend to form aggregates during the milling and drying of the suspensions [12, 21], leading to loss of their large surface area. Soluble polymers and surfactants can be used to impart physical stability and retain the large surface area of the nanoparticles $[1,22,23]$. However, depending on the formulation, drug nanoparticles may not be fully recovered from the dried composites during redispersion and dissolution, which will slow down the drug dissolution and reduce the bioavailability [21, 24]. Here, redispersion testing refers to dispersing the drug composites in various physiologically relevant fluids like water, and comparing the particle size of the resulting suspension with that of the precursor suspension used in drying. Various redispersion methods were studied extensively by Bhakay et al. [21]. Slow/incomplete recovery of drug nanoparticles from the composites was observed when a steric stabilizer or ionic stabilizer was used alone in the precursor drug suspension (e.g. Refs. [12, 21]). Drug particles were fully recovered only when steric stabilizer and ionic stabilizer were used in combination (e.g. Refs. $[9,21])$ or when swellable dispersants were co-milled along with the drug $[13,20]$. However, in all these studies, either single grade (molecular weight) of polymer and/or single polymer concentration was investigated. Molecular weight (MW), a critical property of the polymer, determines both the solution properties of polymer chains and the capability for steric stabilization [25, 26], mechanical properties of films [27], and potentially the release of drug particles for oral administration [28]. Therefore, MW and concentration of polymers may be tailored to achieve the best stabilization performance in milling and fast drug release from the composites. Choi et al. [18] investigated the impact of hydroxypropyl cellulose (HPC) MW on itraconazole nanoparticle production and their recovery from the drug composites, where HPC was used as sole stabilizer at a single concentration, and dissolution performance was not investigated. Sepassi et al. [29] investigated the impact of 
hydroxypropylmethyl cellulose (HPMC) and polyvinylpyrrolidone (PVP) MW on the particle size of milled nabumetone and halofantrine suspensions; however, drying, redispersion, and drug dissolution were not studied. In addition, in the previous works mentioned above, an optimal polymer concentration that ensures full recovery of drug nanoparticles from the composites with minimal usage has not been identified.

To authors' best knowledge, no systematic and comprehensive study has been conducted to elucidate the impact of polymer concentration and molecular weight in the presence/absence of an ionic surfactant on the redispersion and drug dissolution from the nanoparticle-laden composites. Current study aims to fill this gap and develop an understanding of the relationships between the physical stability of drug nanosuspension, redispersion, and drug dissolution from the composites. Griseofulvin (GF) suspensions were wet-milled in a stirred media mill and then fluidized-bed dried-coated onto Pharmatose ${ }^{\circledR}$ carrier particles. Three grades of hydroxypropyl cellulose (HPC) having different molecular weights and sodium dodecyl sulfate (SDS) were used as non-ionic, adsorbing polymer and anionic surfactant, respectively. HPC concentrations varied from $0.25 \%$ to $7.5 \%(\mathrm{w} / \mathrm{w})$ for each polymer molecular weight/grade in the absence/presence of SDS. Laser diffraction, SEM, and UV spectroscopy were used to characterize the drug suspensions and the composites. Particle sizes of the redispersion samples were compared with those of the milled suspensions to assess drug nanoparticle recovery from the composites. Drug dissolution from the composites was measured using a USP II paddle apparatus. Besides elucidating the impact of HPC-SDS, this study also attempts to assess the validity of the commonly-held notion that the use of stable wet-milled drug nanosuspensions ensures fast redispersion and drug dissolution from the dried composites. 


\section{Materials and methods}

\subsection{Materials}

GF (BP/EP grade), a poorly water-soluble drug which belongs to Biopharmaceutics Classification System (BCS) Class II, was purchased from Letco Medical (Decatur, AL, USA) and used as-received in the wet media milling experiments. Three grades (SSL, SL, and L) of hydroxypropyl cellulose (HPC) with $\sim 40, \sim 100$, and $\sim 140 \mathrm{kDa}$ molecular weight, respectively, were donated by Nisso America Inc. (New York, NY, USA) and used as model non-ionic, cellulosic polymer which serves as a steric stabilizer during the milling and a film-former during the drying. SDS purchased from Sigma-Aldrich (Milwaukee, WI, USA) was used as a model anionic surfactant that acts as a wetting agent and electrostatic stabilizer. Its critical micelle concentration $(\mathrm{CMC})$ in water is $8.2 \mathrm{mM}(0.24 \% \mathrm{w} / \mathrm{w})$ at ambient temperature [30]. Pharmatose ${ }^{\circledR}$ DCL 11, a grade of lactose monohydrate produced by DMV International (Netherlands), was used as the water-soluble carrier for fluidized bed drying-coating. Wear-resistant yttrium stabilized zirconia beads (Zirmil Y, Saint Gobain ZirPro, Mountainside, NJ, USA), with a median size of $430 \mu \mathrm{m}$, were used as the milling media.

\subsection{Wet stirred media milling process}

The preparation of initial (feed) drug suspensions followed the same procedure used in Afolabi et al. [31]. The formulation of the suspensions is listed in Table 1. Drug concentration was kept at $10 \%$. All concentrations reported here are with respect to deionized water (300 g). In the absence of SDS, only HPC SSL and L grades were investigated in the $0.25-7.5 \%(\mathrm{w} / \mathrm{w})$ concentration range. At SDS loading of $0.05 \%(\mathrm{w} / \mathrm{w})$, HPC concentrations varied from $0.25 \%$ to $7.5 \%(w / w)$ for all three grades. SDS concentration was purposefully selected well below the 
CMC so as to suppress the Ostwald ripening during the storage [32, 33] because the aqueous solubility of GF is order of magnitude smaller below the CMC than that above the CMC [34].

A Microcer wet stirred media mill (Netzsch Fine Particle Technology, LLC, Exton, PA, USA) with $80 \mathrm{ml}$ chamber was used to prepare the drug nanosuspensions. A peristaltic pump (Masterflex L/S Cole-Parmer Company, USA) recirculated the suspension between the holding tank and the milling chamber, while a screen with a $200 \mu \mathrm{m}$ opening kept the zirconia beads in the milling chamber, but allowing the passage of the suspension. The feed suspensions prepared using a shear mixer (Fisher Scientific Laboratory Stirrer, Catalog no. 14-503, Pittsburgh, PA) were poured into the holding tank and milled under the following conditions: bead loading of 50 $\mathrm{ml}$ (bulk), suspension flow rate of $126 \mathrm{ml} / \mathrm{min}$, and rotor speed of $3200 \mathrm{rpm}$ corresponding to a tip speed of $11.7 \mathrm{~m} / \mathrm{s}$. Both the milling chamber and the holding tank were equipped with a chiller (Advantage Engineering, Greenwood, IN, USA) to keep the suspension below $35^{\circ} \mathrm{C}$. The aforementioned processing parameters were selected based on Bilgili and Afolabi [22]. The particle sizes of the final suspensions (after 80 min milling) were determined, and the suspensions were refrigerated at $8{ }^{\circ} \mathrm{C}$ for one day before coating onto Pharmatose ${ }^{\circledR}$.

\subsection{Fluidized bed drying-coating of drug suspensions onto Pharmatose ${ }^{\circledast}$}

The suspensions prepared by wet media milling, also referred to as precursor suspensions, were sprayed onto Pharmatose ${ }^{\circledR}$ carrier particles in a conventional bench-top fluidized bed processor (Mini-Glatt 5, Glatt Air, Ramsey, NJ, USA) with the top spray configuration. The operating conditions were selected based on Bhakay et al. [21]. 100 g Pharmatose ${ }^{\circledR}$ powder with $d_{50}$ and $d_{90}$ values of $123.6 \mu \mathrm{m}$ and $195.2 \mu \mathrm{m}$ was charged in the product bowl and fluidized at an inlet air pressure of 0.4-0.5 bar. After the powder was fluidized, the heater and suspension spray 
were turned on. About $200 \mathrm{~g}$ milled drug suspension was pumped through a peristaltic pump (Masterflex L/S Cole-Parmer Company, USA) at a constant rate of $0.6 \mathrm{ml} / \mathrm{min}$. Potential sedimentation of the particles was prevented by constant stirring of the suspension during the spraying process. The suspensions were atomized through a bi-fluid nozzle with $0.5 \mathrm{~mm}$ tip diameter at an atomization air pressure of 1 bar. The fluidizing air temperature was set at $70{ }^{\circ} \mathrm{C}$. The coated powder continued to fluidize and dried for $10 \mathrm{~min}$ after all suspension was sprayed. The coated powders (composites) were then tested for particle size and morphology, and used in redispersion-dissolution tests.

\subsection{Particle size measurement}

Particle size distributions (PSDs) were measured using laser diffraction (LS 13 320, Coulter Beckman, Brea, CA, USA). A polarized intensity differential scattering obscuration water optical model was employed. It was maintained between $40 \%$ and $50 \%$ while the obscuration was maintained below $8 \%$ for all measurements. PSD was computed by the software using the Mie scattering theory. Refractive index values are 1.65 for GF and 1.33 for the measurement medium (deionized water). Prior to the size measurement, $\sim 2 \mathrm{ml}$ samples of the milled suspensions were taken from the outlet of mill chamber and diluted with $8 \mathrm{ml}$ solution of water, HPC, SDS, or HPC-SDS depending on the stabilizer(s) used in the milling experiment. The particle size of the suspensions was also measured at the end of the drying process (about $30 \mathrm{~h}$ after milling) to assess aggregation in the suspensions during the storage-coating period, and this size was referred to as the size of the milled suspension before redispersion.

The particle size of the composite particles produced by the fluidized bed drying was measured by a Rodos/Helos laser diffraction system (Sympatec, NJ, USA) based on Fraunhofer 
theory. Approximately $1 \mathrm{~g}$ of the composites was placed on the sample chute of the Rodos dispersing system. As the sample chute was vibrated to feed the sample at a $50 \%$ setting, a dispersion pressure of 0.1 bar was imposed to suck in falling powder through the sample cell of the Rodos.

\subsection{Determination of drug content in the composite powders}

Maximum theoretical amount of GF in $100 \mathrm{mg}$ composites is estimated to be $15.4 \mathrm{mg}$ disregarding losses during the coating (Run 1). GF solubility in methanol is $3 \mathrm{mg} / \mathrm{ml}[21] .100$ $\mathrm{mg}$ of the nanocomposite particles was dissolved in $20 \mathrm{ml}$ of methanol and sonicated for $30 \mathrm{~min}$ to ensure that all GF had dissolved in methanol. The Pharmatose ${ }^{\circledR}$ particles do not dissolve in methanol and remain suspended. After sonication, they were allowed to sediment overnight, and an aliquot of $100 \mu \mathrm{l}$ was taken from the supernatant. This aliquot was diluted to $10 \mathrm{ml}$ methanol. The absorbance of all the samples was measured at the wavelength of $292 \mathrm{~nm}$ via Ultraviolet (UV) spectroscopy by a UV spectrophotometer (Agilent, Santa Clara, CA, USA). Six replicates from each composite formulation were used to calculate the mean drug content along with the percent relative standard deviation (RSD).

\subsection{Redispersion of the drug composites}

The redispersion method was selected based on previous work [11,21]. $1 \mathrm{~g}$ of the composites was weighed and dispersed in $30 \mathrm{ml}$ deionized water for $2 \mathrm{~min}$. This early time point was purposefully selected because of its good discriminatory power for different formulations. The samples were paddle-stirred with a laboratory stirrer (CAT R18, Scientific Instrument Center Limited, Winchester, UK) at $200 \mathrm{rpm}$ for $2 \mathrm{~min}$ to determine if the GF drug particles are redispersed from the composites fast. The maximum amount of drug that can dissolve in water 
during the redispersion test is very small (e.g. about $0.2 \%$ of GF particles for Run 1). Approximately, $0.5 \mathrm{ml}$ aliquot of redispersed sample was taken while stirring and its particle size

was measured. HPC, SDS, and Pharmatose ${ }^{\circledR}$ are all water-soluble. Hence, unless otherwise indicated, the particle sizes obtained from the laser diffraction were mainly the sizes of GF particles and their clusters. The redispersion response in an aqueous solution of SDS at $0.05 \%$ was also studied for formulations with HPC alone. Upon dissolution of the composites with HPC/SDS, the maximum theoretical SDS concentration in deionized water and $0.05 \%$ aqueous SDS solution would be $0.0026 \%$ and $0.0526 \%$, respectively; the highest HPC concentration would be $0.39 \%$. These very low HPC and SDS concentrations are not expected to significantly change the solubility of GF in deionized water. It is known that GF solubility is only slightly increased by SDS below the CMC [34]. Thus, a dissolution-induced size decrease of the drug particles is not likely during the redispersion test.

\subsection{Dissolution test}

Drug dissolution from the as-received (unmilled) drug, its physical mixtures with Runs 2, 7, and 17 formulations, as well as composites was determined via a Distek 2100C dissolution tester (North Brunswick, NJ, USA) according to the USP II paddle method, following the refs. [11, 20, 21]. The dissolution medium was $1000 \mathrm{ml}$ deionized water that was maintained at $37{ }^{\circ} \mathrm{C}$, and a paddle speed of $50 \mathrm{rpm}$ was used for all runs. Deionized water allowed for good discrimination of different formulations under non-sink conditions. The composites were weighed equivalent to a dose of $8.9 \mathrm{mg}$ of GF. They were poured into the dissolution medium and $4 \mathrm{ml}$ samples were taken out manually at $1,2,5,10,20,30$, and $60 \mathrm{~min}$. These aliquots were filtered with a $0.1 \mu \mathrm{m}$ PVDF membrane type syringe filter before UV spectroscopy measurements to minimize any confounding effect of the undissolved drug aggregates. The amount of GF dissolved in deionized 
water was measured by UV spectroscopy at a wavelength of $296 \mathrm{~nm}$. Deionized water was used as the blank. The drug concentration was determined using a calibration curve of drug concentration vs. absorbance with established linearity $\left(R^{2}=1.0000\right.$ with $\left.\mathrm{p}<0.001\right)$. Based on the method development experiments (not mentioned here for the sake of brevity), no significant interference was observed due to the presence of HPC, SDS, and Pharmatose ${ }^{\circ}$ during the UV measurement of griseofulvin concentration in the dissolution testing.

\subsection{Scanning electron microscopy (SEM)}

SEM images of the milled drug particles, Pharmatose ${ }^{\circledR}$ particles, composite particles, and the dried sample after redispersion were taken with LEO 1530 SVMP (Carl Zeiss, Inc., Peabody, MA, USA). For Pharmatose ${ }^{\circledR}$ particles and the composite particles, a carbon tape was placed on an SEM stub, the particles were then placed on the carbon tape. An aliquot of $1 \mathrm{ml}$ drug suspension sample was diluted to $30 \mathrm{ml}$ de-ionized water, vortex-mixed for $30 \mathrm{~s}$, and mounted on a silicon chip (Ted Pella, Inc., Redding, CA, USA). For redispersed samples, after 2 min paddle stirring, a drop sample was mounted on a silicon chip. The stubs with liquid sample were put in a desiccator and allowed to dry overnight under vacuum. All samples were then sputter coated with carbon using BAL-TEC MED 020 (BAL-TEC AG, Balzers, Switzerland) prior to imaging.

\section{Results and discussion}

\subsection{Formation of drug nanoparticles via wet stirred media milling}

Table 1 presents the formulations of the milled drug (GF) suspensions. The formation of GF nanoparticles was first explored in the absence of any stabilizers (Run 1) and in the presence of an anionic surfactant, SDS (Run 2). These two formulations were used as baseline to assess the 
impact of various stabilizers and physical stability of the resulting suspensions. The impact of HPC concentration was then studied in the absence (Runs 3-12) and presence of $0.05 \%$ SDS (Runs 13-27) using SSL, SL, and L grades of HPC with MW of $\sim 40, \sim 100$, and $\sim 140 \mathrm{kDa}$, respectively.

Fig. 1 presents the PSD of the milled suspensions for Runs 1 and 3-12, where HPC was the sole stabilizer and its concentration varied from 0 to $7.5 \%$ for both SSL and L grades. When no stabilizer was used (Run 1), a coarse suspension with particles in the size range of 1-60 $\mu \mathrm{m}$ was formed, as measured by laser diffraction. On the other hand, SEM images show that the asreceived GF particles (Fig. 2a) were broken into 50-400 nm primary nanoparticles (Fig. 2b) upon milling in Run 1. Clearly, Figs. 1 and 2 suggest that the nanoparticles formed upon breakage of the micron-sized GF particles severely aggregated due to high attractive interparticle forces (van der Waals, hydrophobic forces, etc.). Similar observations were reported by Bilgili et al. [32] and Ain-Ai and Gupta [35] for several wet-milled, poorly water-soluble drugs.

When HPC (SSL and L grades) was used as the sole stabilizer especially at a higher concentration, the PSDs shifted to the finer particles, while showing bi-modality due to presence of aggregates (Fig. 1). Although smaller aggregates were formed upon use of lower MW HPC (SSL grade) as compared with L grade, even 7.5\% HPC SSL was insufficient to stabilize GF nanoparticles. Fig. 3 shows PSDs for all formulations with 0.05\% SDS (below the CMC of SDS). When SDS was used alone at this low concentration (0.05\%, Run 2), a tri-modal PSD was observed. This indicates that $0.05 \%$ SDS alone was not able to stabilize the GF nanoparticles. A fine, uni-modal PSD was attained when HPC and SDS were used in combination. The use of HPC-SDS has synergistic stabilizing effects, as demonstrated on multiple drugs [32]. An increase in HPC concentration led to finer particles and narrower PSDs for the SL and L grades, 
whereas the PSD was less sensitive to the concentration for the SSL grade. SDS acts as a wetting agent, allowing for effective deaggregation of the milled particles and it may impart slight electrostatic stabilization, whereas non-ionic HPC adsorbs on GF particles and sterically stabilize the nanoparticles [22]. An increase in cellulosic polymer concentration is known to increase adsorption onto drug particles, thus offering enhanced steric stability via thicker adsorbed polymer layer [29, 33].

The reproducibility of wet stirred media milling of GF and other BCS Class II drugs has been established in several studies $[22,36]$. In this study, the $d_{50}$ and $d_{90}$ values for the repeat of Run 20 were $0.236 \pm 0.001$ and $0.435 \pm 0.002 \mu \mathrm{m}$, respectively. Compared to $d_{50}=0.230 \pm 0.001$ and $d_{90}$ $=0.443 \pm 0.001 \mu \mathrm{m}$ in Run 20 , the deviations in the repeated milling experiment were less than 3\%. Also, as will be shown below, for most formulations, the suspensions did not exhibit a drastic shift in particle sizes after $30 \mathrm{~h}$ storage (before redispersion samples vs. after milling samples), and any variations could be partly attributed to the handling-sampling of the aggregated suspensions following refrigerated storage.

\subsection{Formation of drug composites via fluidized bed drying of the precursor drug suspensions}

The GF suspensions produced by milling were sprayed onto Pharmatose ${ }^{\circledR}$ carrier particles (see Fig. 4a), which form the core of the composite particles, in a fluidized bed drier. Throughout the rest of the paper, all composites are labeled based on the formulation of the respective precursor drug suspension that was used as the feed during the drying. The mean drug content was in the range of $9.3-15.2 \%$ due to different stabilizer concentrations in the milled suspensions and variable drug/Pharmatose ${ }^{\circledR}$ losses to the filters and side walls of the drier (Table 1). Most importantly, the composite powders exhibited pharmaceutically acceptable content uniformity: RSD was less than $6 \%$ for most formulations. Upon atomization, droplets of the milled 
suspensions impinged on Pharmatose ${ }^{\circledR}$ carrier particles and dried, leading to the formation of a single coated composite particle (Fig. 4b), which embeds GF nanoparticles on its shell (Fig. 4c). Some SEM images (not shown for brevity) indicated presence of granules, i.e., doublets, triplets, etc. at higher HPC L concentration, which is quantified by the presence of much coarser

composite particles (see Table 2) than the uncoated Pharmatose ${ }^{\circledR}$ particles with $d_{50}: 123.6 \pm 0.86$ $\mu \mathrm{m}, d_{90}: 195.2 \pm 4.79 \mu \mathrm{m}$. The larger composite particles prepared at higher HPC concentration, especially with the use of $\mathrm{L}$ grade, can be explained by higher viscosity of the respective drug suspensions and coarser droplets formed upon atomization as well as higher coalescence probability of wetted Pharmatose ${ }^{\circledR}$ particles $[21,37]$. SEM images of the composite particle surfaces with different suspension formulations were shown in Fig. 4c-g. Drug aggregates as well as primary nanoparticles encapsulated in the HPC film can be observed on the composite surface.

\subsection{Recovery of the drug nanoparticles from the composite particles in deionized water}

The composite particles produced via fluidized bed drying-coating of precursor GF suspensions onto Pharmatose ${ }^{\circledR}$ were redispersed in water to study the recovery of GF nanoparticles via gentle paddle stirring for $2 \mathrm{~min}$. Fig. 5 presents the particle size statistics of drug particles right after milling, before redispersion ( $30 \mathrm{~h}$ storage after milling), and after redispersion in deionized water for formulations without stabilizers and those with HPC alone having different MWs. Fig. 5 shows that the particle sizes after the redispersion test were much greater than the drug particle sizes after milling and $30 \mathrm{~h}$ storage. Although there were some micron-sized particles in the milled suspensions (Fig. 1), the clusters in the redispersed suspension samples had particle sizes above $10 \mu \mathrm{m}$ (Fig. 5), which are all larger than those in the 
milled suspensions, indicating poor redispersibility of the composites and potentially formation of large clusters during the drying. In fact, it is likely that the shell of some composite particles did not break at all, keeping the core (Pharmatose ${ }^{\circledR}$ ) intact for low HPC concentrations, and that coarse clusters might have appeared after redispersion. A higher polymer concentration (especially at and above $2.5 \%$ ) led to smaller clusters after redispersion for both HPC grades.

A specific example of the poor redispersion is the composite particles with no stabilizer in the suspension (Run 1), which had the median size of $127.8 \mu \mathrm{m}$ (Table 2). After redispersion in deionized water, the median size was $105.5 \mu \mathrm{m}$, whereas the milled suspension had GF median size of $9.1 \mu \mathrm{m}$ (Fig. 5). SEM images of the redispersed suspension samples following drying are shown in Fig. 6. Fig. 6a confirmed the aforementioned poor redispersion: the Run 1 composite particles slightly shrunk upon partial dissolution of Pharmatose ${ }^{\circledR}$ and the coated layer (shell structure) broke into large pieces. The poor redispersion is most likely due to hard aggregates formed upon removal of water during the drying; solid bridges could have formed upon recrystallization of the small amount of dissolved GF. The hard aggregates might not have allowed for proper shell breakage and or full dispersion into primary GF nanoparticles. Moreover, without having any stabilizers, Run 1 composite particles are covered solely by hard aggregates of hydrophobic GF particles, which may have slowed down water penetration into the core. During the redispersion test, water penetrates through the pores in the shell of the composite particles and dissolves the Pharmatose ${ }^{\circledR}$ core, which is largely inhibited by the poor wettability of Run 1 composite, leading to the coarse clusters (Fig. 6a).

For composites with HPC as the sole stabilizer, HPC acts as a film former and partially covers hydrophobic GF nanoparticles (e.g. Fig. 4d). Due to the dissolution of HPC and the mechanical action of paddle stirring, the shell of the composite particles broke down to smaller 
clusters in water, especially at higher HPC concentration as compared with Run 1 case (Figs. 5 and $6 \mathrm{~b}$ ). On the other hand, even with $7.5 \% \mathrm{HPC}$, the primary drug nanoparticles cannot be recovered for both HPC grades. This is partly due to the slow dissolution of HPC and its relatively low wetting capability [38, 39], which limited the water penetration. Moreover, the hydrophobic nature of GF led to poor wetting of the clusters, thus slowing down the release of drug nanoparticles. Finally, the precursor suspensions with HPC as the sole stabilizer exhibited severe aggregation (see Fig. 5). Hence, the redispersion of these composites at best might have resulted in aggregates whose sizes would be similar to those in the precursor suspensions; however, much larger clusters formed due to the poor wettability and the formation of hard aggregates especially below $2.5 \%$ HPC.

Fig. 7 shows the particle size statistics for the composites with SDS and HPC of various MWs after redispersion and those of the corresponding precursor suspensions. As a baseline, Run 2 had only SDS in the precursor suspension. Drug nanoparticles in the milled suspension shows significant particle size increase after $30 \mathrm{~h}$ storage (before redispersion) and drug nanoparticles were not recovered from its dried composite. SDS can impart excellent wettability and thus effective deaggregation of clusters that formed during or after the breakdown of the shell under the action of gentle paddle stirring [21]. However, SDS alone was not able to provide sufficient coverage of the drug nanoparticles and prevent the formation of hard aggregates during drying. This, coupled with the presence of aggregates formed during the storage, could explain the poor redispersibility of Run 2 composite without any HPC. It appears that while wettability imparted by SDS and small aggregates formed during the milling upon use of SDS are both desirable, a minimum HPC concentration is still required.

The formulations with SDS (Fig. 7) exhibited smaller drug particle sizes and lower extent of 
drug nanoparticle aggregation in the milled suspensions, enhanced wettability of the composites, and faster redispersion and recovery of the drug nanoparticles as compared with those without SDS (Fig. 5), pointing out the criticality of SDS. Moreover, with $0.05 \%$ SDS, an increase in HPC concentration led to smaller milled particle sizes and faster/more complete recovery of the drug nanoparticles (Fig. 7). Different from the formulations with HPC alone (Fig. 5), the formulations with SDS led to effective recovery of the drug nanoparticles at 5\% HPC SSL or $2.5 \%$ HPC SL or L. The SEM image (Fig. 6d) qualitatively confirms the full recovery of nanoparticles from the composite with SDS. The excellent wettability and water penetration imparted by SDS led to the fast disintegration of the shell of the composites followed by fast dispersion of any clusters into drug nanoparticles. However, despite ensuring the formation of fine nanoparticles in the respective milled suspensions, the use of SDS did not allow for full nanoparticle recovery when HPC was below a threshold concentration $(\sim 1 \%)$ (see e.g. Fig. 6c). This may be explained by the formation of hard aggregates during the drying process when HPC concentration was low. Considering that the respective milled suspensions with SDS had low extent of aggregation and were relatively stable, the existence of large clusters emanating from the redispersed composites (Fig. 7) implies that a relatively stable nanosuspension did not guarantee full and fast recovery of nanoparticles from the composites. It is concluded that the presence of sufficient amount of HPC is critical for full recovery of drug nanoparticles, and a lower HPC concentration may be sufficient for the higher molecular weight (SL or L grades).

\subsection{Recovery of drug nanoparticles from the drug composite particles in $0.05 \%$ SDS solution}

To further elucidate the role of SDS and investigate the impact of wettability of the composites, the composites with HPC alone (without SDS) were redispersed in $0.05 \%$ SDS 
solution (see Fig. 8), in comparison to their redispersion in de-ionized water (Fig. 5). This medium has the same SDS concentration as in the milled suspensions containing SDS (Fig. 7). It should be noted that GF solubility is only slightly increased by SDS below the CMC [34]. Interestingly, drug nanoparticles were effectively recovered only from the composites with high HPC concentration, at or above 5\%, for both SSL and L grades (Fig. 8) because $0.05 \%$ SDS in the redispersion medium enhanced the wetting and dispersed the drug aggregates, unlike the poor redispersion in de-ionized water (Fig. 5). Drug nanoparticles could not be recovered from the composites with less than 5\% HPC despite the presence of $0.05 \%$ SDS in the redispersion medium. Figs. 5 and 8 both illustrate coarse clusters $(>10 \mu \mathrm{m})$ at or below $1 \%$ HPC. These results can again be explained by the presence of significant fraction of aggregates in the composites without SDS (refer to Fig. 1) and formation of hard aggregates during drying, which did not allow for fast shell breakage/disintegration even under paddle stirring with good wettability imparted by SDS. Similar results were obtained by de Villiers for aggregated drug powders [40]. High HPC concentration (>2.5\%) completely prevents the formation of hard aggregates during drying and allows for proper film formation in the shell of the composite particle. The $0.05 \%$ SDS solution provides sufficient wetting to the composites and allows for more effective deaggregation than deionized water. Due to the prevention of hard aggregates formation at high HPC concentration and sufficient wetting imparted by $0.05 \%$ SDS solution, even some of the aggregates formed in the precursor drug suspensions without SDS was effectively deaggregated. Hence, for composites with high HPC concentration $(>2.5 \%)$, the particle sizes after redispersion in the $0.05 \%$ SDS solution were smaller than those in the respective precursor suspensions without SDS after milling/before redispersion (Fig. 8). 
A comparison of the redispersed particle sizes for the formulations with $0.05 \%$ SDS in the respective precursor suspensions, which led to an estimated $0.0026 \%$ SDS upon the redispersion of the respective composites in de-ionized water (see Fig. 7a,c), vs. those formulations with HPC/without SDS, redispersed in $0.05 \%$ SDS solution (see Fig. 8a,b) sheds further insight. Despite the attainment of $0.0026 \%$ SDS concentration in the redispersion medium, $0.05 \%$ SDS in the milled suspension/composite led to smaller redispersed particles, i.e., more effective recovery of the GF nanoparticles, notably at the intermediate HPC concentrations, i.e., $1 \%$ and 2.5\% HPC (Fig. 7), as compared with $0.05 \%$ SDS added to the redispersion medium directly (Fig. 8). A similar, albeit less pronounced difference occurred at 0 and $0.25 \%$ HPC because hard aggregate formation controlled the poor redispersibility observed at such low HPC concentration. These findings could be mainly explained by the presence of smaller aggregates in the precursor milled suspensions with SDS (refer to Fig. 3) than without SDS (refer to Fig. 1) besides the facilitated disintegration of the composite shell with enhanced wettability during the redispersion test. At 5\% and 7.5\% HPC, a similar pattern was observed for the L grade, whereas an anomaly occurred for the SSL grade for which the smaller redispersed particles were obtained when $0.05 \%$ SDS was added to the redispersion medium directly. It seems the much higher SDS concentration, i.e., $0.05 \%$ vs. $0.0026 \%$, in the redispersion medium affected the redispersion results differently only for the high HPC SSL concentration.

Overall, the redispersion experiments with deionized water and $0.05 \%$ SDS solution as two separate media point out the need for preparing aggregate-free precursor (milled) drug suspensions upon use of HPC-SDS combination. Moreover, a minimum HPC concentration (within $0.25-1 \%$ in the precursor) is required to prevent hard-aggregate formation during drying and to give strength and proper film formation in the shell of the composite particles. 


\subsection{Drug dissolution from the composites}

The as-received (unmilled) drug, its physical mixtures with Runs 2, 7, and 17 formulations, as well as composites produced via fluidized bed drying were dissolved in excess de-ionized water to allow for full dissolution of the poorly water-soluble drug. Dissolution profiles of the composites without SDS in the precursor suspensions are shown in Fig. 9. For both HPC SSL and $\mathrm{L}$, dissolution rates increased with higher polymer concentration, but immediate drug release (80\% dissolved within $20 \mathrm{~min}$ ) was not realized for any formulation. These findings can overall be explained by the poor redispersibility of the composites without SDS (refer to Fig. 5) and slow/incomplete nanoparticle recovery during the dissolution. It is expected that GF nanoparticle recovery precedes GF dissolution from the composites considering the poorly water-soluble nature of this drug. Also note that since a coarse GF suspension with large aggregates in the size range of 1-60 $\mu \mathrm{m}$ was formed in the absence of stabilizers (refer to Section 3.1), the slowest GF dissolution occurred from the composites with no HPC/SDS, signifying the criticality of adequate stabilization of the precursor drug suspension as a necessary condition.

For HPC SSL at the highest concentration (7.5\%), the dissolution of drug particles only reached $50.2 \%$ at $60 \mathrm{~min}$. Applying $f_{1}$ and $f_{2}$ tests and $t$-test $[41,42]$, we found that the dissolution profiles of the composite formulations with 1-7.5\% HPC SSL presented in Fig. 9 were not statistically different. For $7.5 \%$ HPC L having the highest polymer molecular weight, the drug dissolution reached $68.8 \%$, which is higher than that for HPC SSL at the same concentration. This may be due to the better coverage of the hydrophobic drug surfaces with higher MW HPC, despite its slower diffusion/dissolution in water. It should be noted that, in the absence of SDS, HPC as a film former in composites can only provide limited wettability for dissolution, which is the limiting mechanism for their dissolution performance. 
Dissolution profiles of the composites with $0.05 \%$ SDS in the precursor suspensions are shown in Fig. 10. A comparative analysis of the results in Figs. 9 and 10 suggests that most composites with SDS exhibited immediate drug release and faster drug dissolution than those without SDS, which can be overall explained by the enhanced redispersibility/faster nanoparticle recovery (refer to Fig. 7) stemming from the excellent wettability and synergistic stabilization imparted by the use of HPC-SDS. An increase in HPC concentration led to faster dissolution for all three HPC grades. This positive effect of HPC appears to saturate when the HPC concentration was above $2.5 \%$ for SSL and SL grades and 5\% for L grade. These observations can again be explained by enhanced nanoparticle recovery (smaller redispersed particle sizes) at higher HPC concentration and the tendency of the redispersed particle sizes to minimum values above 2.5\% HPC (refer to Fig. 7). At or below 5\% HPC concentration, unlike the formulations without SDS, the formulations with HPC-SDS exhibited slower dissolution when a higher MW HPC was used. With SDS, the dissolution profiles are more sensitive to the dissolution-diffusion rate of HPC with various MW. The slower dissolution for the composites with higher MW HPC may be explained by the slower dissolution and diffusion of the higher MW HPC. In addition, the relatively large granules formed during the drying process for the higher MW HPC (L grade in Table 2) may also partly explain the slower dissolution profiles.

Additional insight into GF dissolution from the composites can be gained by analyzing the dissolution performance of as-received (unmilled) GF alone and its physical mixtures with Runs 2, 7, and 17 formulations. Unmilled GF exhibited $16.8 \pm 4.2 \%$ release after $60 \mathrm{~min}$. To investigate the net effect of HPC and SDS, physical mixtures of GF in Runs 2 and 7 formulations were prepared and dissolved separately. Run 2 physical mixture had unmilled griseofulvin, SDS, and Pharmatose ${ }^{\circledR}$; whereas Run 7 physical mixture had the highest amount of HPC (SSL grade) 
with unmilled griseofulvin and Pharmatose ${ }^{\circledR}$. Runs 2 and 7 physical mixtures exhibited GF release of $20.9 \pm 0.6 \%$ and $38.3 \pm 0.4 \%$, respectively. Run 17 physical mixture had unmilled drug and all excipients, i.e., HPC, SDS, and Pharmatose ${ }^{\circledR}$. Simply mixing unmilled drug with HPC, SDS, and Pharmatose ${ }^{\circledR}$ even at the highest HPC concentration used (Run 17) did not lead to fast, immediate drug release: $41.7 \pm 0.7 \%$ after $60 \mathrm{~min}$, as compared with $100 \%$ for Run 17 composites (see Fig. 10a). For the unmilled drug, the addition of either HPC or SDS improved the dissolution performance; while this effect appears to be simply additive, i.e., no synergy exhibited by Run 17 physical mixture. On the other hand, comparative analysis of the drug release data at 60 min for Runs 2, 7, and 17 composites (see Figs. 9a and 10a) suggests a synergistic effect of the HPC-SDS combination for the composites, which is in line with the synergistic stabilization effects mentioned in Section 3.1. Apparently, the finer nanoparticles in the precursor suspensions, which result from this synergistic stabilization of the nanosuspensions via HPC-SDS combination, enhanced the drug dissolution performance of the composites. Finally, the dissolution performances of the physical mixtures were inferior to those of the corresponding composites prepared via wet-milling followed by fluidized bed drying-coating (Figs. 9a and 10a). This finding reveals the significant role that wet media milling plays in generating drug nanoparticles and enhancing the drug surface area available for dissolution from the composites.

\section{Conclusions}

Nanocomposite microparticles were produced by fluidized bed drying-coating of wet stirred media-milled GF nanosuspension onto Pharmatose ${ }^{\circledR}$ carrier particles. The drug composites were redispersed in water and aqueous solution of SDS. Three different HPC polymer molecular 
weights at varied concentrations in the absence/presence of SDS were investigated for their ability to enhance the redispersion and dissolution of the drug nanoparticles. The results overall suggest that well-stabilized drug nanosuspensions with minimal amount of aggregates is necessary to produce drug composites with good redispersion and fast dissolution. However, good physical stability and prevention of aggregation in the milled suspensions is not sufficient. The presence of a soluble, adsorbing polymer above a minimum concentration is also needed so as to suppress formation of hard aggregates during drying. Hence, our study concludes that proper stabilization of drug suspensions is a necessary condition for fast drug nanoparticles recovery and fast drug dissolution from the composites, but not sufficient. Sufficiency originates from the use of SDS in combination with HPC above the threshold concentration, which results in almost aggregate-free drug nanosuspensions and mitigates hard-aggregate formation. Immediate drug release is achieved when a lower molecular weight (SSL grade) HPC at or above $1 \%$ concentration was used along with $0.05 \%$ SDS. Despite the use of $0.05 \%$ SDS, the highest grade of HPC (L grade) entails relatively high concentrations to ensure immediate drug release from the composites.

\section{Acknowledgements}

The authors report financial support through Grant EEC-0540855 from the U.S. National Science Foundation (NSF) Engineering Research Center (ERC) for Structured Organic Particulate Systems (SOPS). Nisso America Inc. is also noted for the kind donation of the HPC samples. Critical review and comments by Dr. M. Azad of MIT are gratefully acknowledged. 


\section{References}

[1] F. Kesisoglou, S. Panmai, Y. Wu, Nanosizing-oral formulation development and biopharmaceutical evaluation, Advanced Drug Delivery Reviews, 59 (2007) 631-644.

[2] S.K. Singh, K. Srinivasan, K. Gowthamarajan, D.S. Singare, D. Prakash, N.B. Gaikwad, Investigation of preparation parameters of nanosuspension by top-down media milling to improve the dissolution of poorly water-soluble glyburide, European Journal of Pharmaceutics and Biopharmaceutics, 78 (2011) 441-446.

[3] Y. Tanaka, M. Inkyo, R. Yumoto, J. Nagai, M. Takano, S. Nagata, Nanoparticulation of probucol, a poorly water-soluble drug, using a novel wet-milling process to improve in vitro dissolution and in vivo oral absorption, Drug Development and Industrial Pharmacy, 38 (2012) 1015-1023.

[4] A.A. Noyes, W.R. Whitney, The rate of solution of solid substances in their own solutions, Journal of the American Chemical Society, 19 (1897) 930-934.

[5] R.H. Müller, S. Gohla, C.M. Keck, State of the art of nanocrystals - Special features, production, nanotoxicology aspects and intracellular delivery, European Journal of Pharmaceutics and Biopharmaceutics, 78 (2011) 1-9.

[6] B. Sun, Y. Yeo, Nanocrystals for the parenteral delivery of poorly water-soluble drugs, Current Opinion in Solid State and Materials Science, 16 (2012) 295-301.

[7] E. Merisko-Liversidge, G.G. Liversidge, Nanosizing for oral and parenteral drug delivery: A perspective on formulating poorly-water soluble compounds using wet media milling technology, Advanced Drug Delivery Reviews, 63 (2011) 427-440.

[8] C.M. Keck, R.H. Müller, Drug nanocrystals of poorly soluble drugs produced by high pressure homogenisation, European Journal of Pharmaceutics and Biopharmaceutics, 62 (2006) 3-16.

[9] S. Basa, T. Muniyappan, P. Karatgi, R. Prabhu, R. Pillai, Production and in vitro characterization of solid dosage form incorporating drug nanoparticles, Drug Development and Industrial Pharmacy, 34 (2008) 1209-1218.

[10] B. Van Eerdenbrugh, L. Froyen, J. Van Humbeeck, J.A. Martens, P. Augustijns, G. Van den Mooter, Drying of crystalline drug nanosuspensions - the importance of surface hydrophobicity on dissolution behavior upon redispersion, European Journal of Pharmaceutical Sciences, 35 (2008) 127-135.

[11] A. Bhakay, M. Azad, E. Bilgili, R. Dave, Redispersible fast dissolving nanocomposite microparticles of poorly water-soluble drugs, International Journal of Pharmaceutics, 461 (2014) 367-379.

[12] J. Lee, Drug nano - and microparticles processed into solid dosage forms: physical properties, Journal of Pharmaceutical Sciences, 92 (2003) 2057-2068.

[13] M. Azad, C. Arteaga, B. Abdelmalek, R. Davé, E. Bilgili, Spray drying of drug-swellable dispersant suspensions for preparation of fast-dissolving, high drug-loaded, surfactant-free nanocomposites, Drug Development and Industrial Pharmacy, 41 (2015) 1617-1631.

[14] W.S. Cheow, M.L.L. Ng, K. Kho, K. Hadinoto, Spray-freeze-drying production of thermally sensitive polymeric nanoparticle aggregates for inhaled drug delivery: effect of freeze-drying adjuvants, International Journal of Pharmaceutics, 404 (2011) 289-300.

[15] Y. Wang, K. Kho, W.S. Cheow, K. Hadinoto, A comparison between spray drying and spray freeze drying for dry powder inhaler formulation of drug-loaded lipid-polymer hybrid nanoparticles, International Journal of Pharmaceutics, 424 (2012) 98-106.

[16] A.M. Layre, P. Couvreur, J. Richard, D. Requier, N. Eddine Ghermani, R. Gref, Freeze-Drying of Composite Core-Shell Nanoparticles, Drug Development \& Industrial Pharmacy, 32 (2006) 839-846.

[17] H. de Waard, W.L.J. Hinrichs, H.W. Frijlink, A novel bottom-up process to produce drug nanocrystals: Controlled crystallization during freeze-drying, Journal of controlled release, 128 (2008) 179-183.

[18] J.-Y. Choi, C.H. Park, J. Lee, Effect of polymer molecular weight on nanocomminution of poorly soluble drug, Drug Delivery, 15 (2008) 347-353. 
[19] S. Kim, J. Lee, Effective polymeric dispersants for vacuum, convection and freeze drying of drug nanosuspensions, International Journal of Pharmaceutics, 397 (2010) 218-224.

[20] A. Bhakay, M. Azad, E. Vizzotti, R.N. Dave, E. Bilgili, Enhanced recovery and dissolution of griseofulvin nanoparticles from surfactant-free nanocomposite microparticles incorporating wetmilled swellable dispersants, Drug Development and Industrial Pharmacy, 40 (2014) 1509-1522.

[21] A. Bhakay, R. Davé, E. Bilgili, Recovery of BCS Class II drugs during aqueous redispersion of core-shell type nanocomposite particles produced via fluidized bed coating, Powder Technology, 236 (2013) 221-234.

[22] E. Bilgili, A. Afolabi, A combined microhydrodynamics-polymer adsorption analysis for elucidation of the roles of stabilizers in wet stirred media milling, International Journal of Pharmaceutics, 439 (2012) 193-206.

[23] A. Bhakay, M. Merwade, E. Bilgili, R.N. Dave, Novel aspects of wet milling for the production of microsuspensions and nanosuspensions of poorly water-soluble drugs, Drug Development and Industrial Pharmacy, 37 (2011) 963-976.

[24] M.V. Chaubal, C. Popescu, Conversion of nanosuspensions into dry powders by spray drying: a case study, Pharmaceutical research, 25 (2008) 2302-2308.

[25] H. Ploehn, W. Russel, Interactions between colloidal particles and soluble polymers, Advances in chemical engineering, 15 (1990) 137-228.

[26] A. Adamson, A. Gast, Physical chemical of surfaces, in, John Wiley \& Son, Inc., New York, 1997.

[27] R. Rowe, The effect of the molecular weight of ethyl cellulose on the drug release properties of mixed films of ethyl cellulose and hydroxypropylmethylcellulose, International Journal of Pharmaceutics, 29 (1986) 37-41.

[28] G. Mittal, D.K. Sahana, V. Bhardwaj, M.N.V. Ravi Kumar, Estradiol loaded PLGA nanoparticles for oral administration: Effect of polymer molecular weight and copolymer composition on release behavior in vitro and in vivo, Journal of controlled release, 119 (2007) 77-85.

[29] S. Sepassi, D.J. Goodwin, A.F. Drake, S. Holland, G. Leonard, L. Martini, M.J. Lawrence, Effect of polymer molecular weight on the production of drug nanoparticles, Journal of Pharmaceutical Sciences, 96 (2007) 2655-2666.

[30] K. Kalyanasundaram, J.K. Thomas, Environmental effects on vibronic band intensities in pyrene monomer fluorescence and their application in studies of micellar systems, Journal of the American Chemical Society, 99 (1977) 2039-2044.

[31] A. Afolabi, O. Akinlabi, E. Bilgili, Impact of process parameters on the breakage kinetics of poorly water-soluble drugs during wet stirred media milling: A microhydrodynamic view, European Journal of Pharmaceutical Sciences, 51 (2014) 75-86.

[32] E. Bilgili, M. Li, A. Afolabi, Is the combination of cellulosic polymers and anionic surfactants a good strategy for ensuring physical stability of BCS Class II drug nanosuspensions?, Pharmaceutical Development and Technology, 21 (2016) 499-510.

[33] C. Knieke, M. Azad, R. Davé, E. Bilgili, A study of the physical stability of wet media-milled fenofibrate suspensions using dynamic equilibrium curves, Chemical Engineering Research and Design, 91 (2013) 1245-1258.

[34] V.M. Rao, M. Lin, C.K. Larive, M.Z. Southard, A mechanistic study of griseofulvin dissolution into surfactant solutions under laminar flow conditions, Journal of Pharmaceutical Sciences, 86 (1997) $1132-1137$.

[35] A. Ain-Ai, P.K. Gupta, Effect of arginine hydrochloride and hydroxypropyl cellulose as stabilizers on the physical stability of high drug loading nanosuspensions of a poorly soluble compound, International Journal of Pharmaceutics, 351 (2008) 282-288.

[36] M. Li, N. Yaragudi, A. Afolabi, R. Dave, E. Bilgili, Sub-100nm drug particle suspensions prepared via wet milling with low bead contamination through novel process intensification, Chemical Engineering Science, 130 (2015) 207-220. 
[37] E. Bilgili, L.A. Rosen, J.S. Ko, A. Chen, E.J. Smith, K. Fliszar, G. Wong, Experimental study of fluidized bed co-granulation of two active pharmaceutical ingredients: an industrial scale-up perspective, Particulate Science and Technology, 29 (2011) 285-309.

[38] N. Rasenack, H. Hartenhauer, B.W. Müller, Microcrystals for dissolution rate enhancement of poorly water-soluble drugs, International Journal of Pharmaceutics, 254 (2003) 137-145.

[39] S.V. Dalvi, R.N. Dave, Analysis of nucleation kinetics of poorly water-soluble drugs in presence of ultrasound and hydroxypropyl methyl cellulose during antisolvent precipitation, International Journal of Pharmaceutics, 387 (2010) 172-179.

[40] M.M. de Villiers, Influence of agglomeration of cohesive particles on the dissolution behaviour of furosemide powder, International Journal of Pharmaceutics, 136 (1996) 175-179.

[41] M. Azad, A. Afolabi, A. Bhakay, J. Leonardi, R. Davé, E. Bilgili, Enhanced physical stabilization of fenofibrate nanosuspensions via wet co-milling with a superdisintegrant and an adsorbing polymer, European Journal of Pharmaceutics and Biopharmaceutics, 94 (2015) 372-385.

[42] J.W. Moore, H.H. Flanner, Mathematical comparison of dissolution profiles, Pharmaceutical Technology, 20 (1996) 64-74. 


\section{List of table captions}

Table 1. Formulations of the milled drug suspensions and drug content of the composites.

Table 2. Particle sizes of the composites prepared using various precursor suspensions. 


\section{Table 1.}

Formulations of the milled drug suspensions and drug content of the composites.

\begin{tabular}{|c|c|c|c|c|}
\hline \multirow[b]{2}{*}{$\begin{array}{l}\text { Run } \\
\text { No. }\end{array}$} & \multirow[b]{2}{*}{$\begin{array}{l}\text { HPC } \\
\text { grade }\end{array}$} & \multicolumn{2}{|c|}{ Suspension } & \multirow{2}{*}{$\begin{array}{c}\text { Composite } \\
\text { Average, RSD } \\
(\% \text { w/w, \% })^{b}\end{array}$} \\
\hline & & $\begin{array}{c}\text { HPC } \\
(\% \mathrm{w} / \mathrm{w})^{\mathrm{a}}\end{array}$ & $\begin{array}{c}\text { SDS } \\
(\% \mathrm{w} / \mathrm{w})^{\mathrm{a}}\end{array}$ & \\
\hline 1 & - & - & - & $15.2,5.95$ \\
\hline 2 & - & - & 0.05 & $13.8,7.28$ \\
\hline 3 & SSL & 0.25 & 0 & $15.0,8.08$ \\
\hline 4 & SSL & 1 & 0 & $14.9,3.36$ \\
\hline 5 & SSL & 2.5 & 0 & $12.5,5.74$ \\
\hline 6 & SSL & 5 & 0 & $14.0,4.61$ \\
\hline 7 & SSL & 7.5 & 0 & $12.5,5.19$ \\
\hline 8 & $\mathrm{~L}$ & 0.25 & 0 & $11.3,1.72$ \\
\hline 9 & $\mathrm{~L}$ & 1 & 0 & $12.3,2.16$ \\
\hline 10 & $\mathrm{~L}$ & 2.5 & 0 & $13.7,1.12$ \\
\hline 11 & $\mathrm{~L}$ & 5 & 0 & $11.7,2.09$ \\
\hline 12 & $\mathrm{~L}$ & 7.5 & 0 & $10.3,4.67$ \\
\hline 13 & SSL & 0.25 & 0.05 & $12.1,3.17$ \\
\hline 14 & SSL & 1 & 0.05 & $13.2,0.16$ \\
\hline 15 & SSL & 2.5 & 0.05 & $13.5,6.21$ \\
\hline 16 & SSL & 5 & 0.05 & $12.8,1.31$ \\
\hline 17 & SSL & 7.5 & 0.05 & $11.6,5.02$ \\
\hline 18 & $\mathrm{SL}$ & 0.25 & 0.05 & $13.5,5.64$ \\
\hline 19 & SL & 1 & 0.05 & $11.5,5.71$ \\
\hline 20 & SL & 2.5 & 0.05 & $11.3,5.95$ \\
\hline 21 & SL & 5 & 0.05 & $10.6,3.21$ \\
\hline 22 & SL & 7.5 & 0.05 & $11.2,6.30$ \\
\hline 23 & $\mathrm{~L}$ & 0.25 & 0.05 & $12.1,4.08$ \\
\hline 24 & $\mathrm{~L}$ & 1 & 0.05 & $14.3,0.69$ \\
\hline 25 & $\mathrm{~L}$ & 2.5 & 0.05 & $11.9,2.88$ \\
\hline 26 & $\mathrm{~L}$ & 5 & 0.05 & $12.0,2.29$ \\
\hline 27 & $\mathrm{~L}$ & 7.5 & 0.05 & $9.30,6.43$ \\
\hline
\end{tabular}

${ }^{\mathrm{a}} \mathrm{w} / \mathrm{w}$ with respect to deionized water in the suspension.

${ }^{\mathrm{b}} \mathrm{w} / \mathrm{w}$ with respect to dried composite. 


\section{Table 2.}

Particle sizes of the composites prepared using various precursor suspensions.

\begin{tabular}{lcccc}
\hline & \multicolumn{4}{c}{ Drug composite particle size $(\mu \mathrm{m})$} \\
\cline { 2 - 5 } HPC concentration & \multicolumn{2}{c}{ Without SDS } & \multicolumn{2}{c}{ With 0.05\% SDS } \\
\cline { 2 - 5 }$(\%$ w/w), grade & $d_{50} \pm$ SD & $d_{90} \pm$ SD & $d_{50} \pm$ SD & $d_{90} \pm$ SD \\
\hline $0 \%,-$ & $127.8 \pm 0.67$ & $201.8 \pm 3.61$ & $130.1 \pm 2.34$ & $199.1 \pm 6.72$ \\
$0.25 \%$, SSL & $123.2 \pm 0.75$ & $187.7 \pm 2.58$ & $123.4 \pm 2.17$ & $199.0 \pm 8.25$ \\
$1 \%$, SSL & $124.1 \pm 2.33$ & $193.2 \pm 3.08$ & $124.6 \pm 1.79$ & $196.4 \pm 4.36$ \\
$2.5 \%$, SSL & $124.6 \pm 3.94$ & $199.4 \pm 7.63$ & $127.4 \pm 3.24$ & $201.0 \pm 5.85$ \\
$5 \%$, SSL & $131.2 \pm 2.43$ & $207.7 \pm 7.49$ & $128.6 \pm 1.96$ & $202.8 \pm 4.16$ \\
$7.5 \%$, SSL & $152.9 \pm 5.53$ & $320.5 \pm 25.2$ & $145.3 \pm 0.52$ & $229.1 \pm 6.09$ \\
$0.25 \%, \mathrm{~L}$ & $126.4 \pm 2.02$ & $192.7 \pm 2.20$ & $139.2 \pm 3.00$ & $222.6 \pm 12.5$ \\
$1 \%, \mathrm{~L}$ & $137.7 \pm 0.96$ & $212.5 \pm 1.93$ & $154.3 \pm 3.45$ & $236.4 \pm 7.93$ \\
$2.5 \%, \mathrm{~L}$ & $163.0 \pm 0.29$ & $249.8 \pm 6.17$ & $187.2 \pm 7.21$ & $381.5 \pm 24.6$ \\
$5 \%, \mathrm{~L}$ & $238.4 \pm 2.71$ & $436.0 \pm 26.5$ & $251.1 \pm 6.55$ & $428.8 \pm 18.4$ \\
$7.5 \%, \mathrm{~L}$ & $289.8 \pm 24.1$ & $442.7 \pm 18.5$ & $260.7 \pm 0.61$ & $502.2 \pm 22.3$ \\
\hline
\end{tabular}




\section{Figure captions}

Fig. 1. Volume frequency distributions of various milled drug suspensions containing HPC with different molecular weights: (a) $40 \mathrm{kDa}$ (SSL grade, Runs 3-7) and (b) $140 \mathrm{kDa}$ (L grade, Runs 8-12). Run 1 had no stabilizers.

Fig. 2. SEM images of drug (GF) particles: (a) before milling (marker size: $10 \mu \mathrm{m}, 2 \mathrm{k} \times$ magnification) and (b) after milling (Run 1, without HPC/SDS) (marker size: $200 \mathrm{~nm}, 40 \mathrm{k} \times$ magnification).

Fig. 3. Volume frequency distributions of various milled drug suspensions containing $0.05 \%$ SDS and HPC with different molecular weights: (a) 40 kDa (SSL grade, Runs 13-17), (b) 100 kDa (SL grade, Runs 18-22), and (c) 140 kDa (L grade, Runs 23-27). Run 2 had only $0.05 \%$ SDS.

Fig. 4. SEM images of the surfaces of (a) as-received Pharmatose ${ }^{\circledR}$ particles (marker size: 2 $\mu \mathrm{m}, 3 \mathrm{k} \times$ magnification) as well as the composites prepared using (b) $1 \%$ HPC SSL, $0.05 \%$ SDS (Run 14) (marker size: $2 \mu \mathrm{m}, 4 \mathrm{k} \times$ magnification), (c) 1\% HPC SSL, $0.05 \%$ SDS (Run 14, higher magnification) (marker size: $1 \mu \mathrm{m}, 20 \mathrm{k} \times$ magnification), (d) $1 \%$ HPC SSL (Run 4) (marker size: $1 \mu \mathrm{m}, 4 \mathrm{k} \times$ magnification), (e) 5\% HPC SSL, 0.05\% SDS (Run 16) (marker size: $1 \mu \mathrm{m}, 20 \mathrm{k} \times$ magnification), (f) $1 \%$ HPC L, 0.05\% SDS (Run 24) (marker size: $1 \mu \mathrm{m}$, 20kx magnification), and (g) 5\% HPC L, 0.05\% SDS (Run 26) (marker size: $1 \mu \mathrm{m}, 20 \mathrm{k} \times$ magnification).

Fig. 5. Particle sizes of the composites prepared using various precursor suspensions with (a) HPC SSL (40 kDa, Runs 3-7) and (b) HPC L (140 kDa, Runs 8-12) after 2 min redispersion in deionized water in comparison to the particle sizes of the suspensions after milling and after $30 \mathrm{~h}$ storage (before redispersion). Run 1 had no stabilizers. 
Fig. 6. SEM images of the drug composites prepared using various precursor suspensions (a) without stabilizers (Run 1) (marker size: $10 \mu \mathrm{m}, 3 \mathrm{k} \times$ magnification) as well as with (b) $7.5 \%$ HPC SSL (Run 7) (marker size: $10 \mu \mathrm{m}, 500 \times$ magnification), (c) $0.25 \%$ HPC SSL, $0.05 \%$ SDS (Run 13) (marker size: $10 \mu \mathrm{m}, 1 \mathrm{k} \times$ magnification), and (d) 5\% HPC SSL, $0.05 \%$ SDS (Run 16) (marker size: $200 \mathrm{~nm}, 40 \mathrm{k} \times$ magnification) after 2 min redispersion in deionized water.

Fig. 7. Particle sizes of the composites prepared using precursor suspensions with $0.05 \%$ SDS and (a) HPC SSL (40 kDa, Runs 13-17), (b) HPC SL (100 kDa, Runs 18-22), and (c) HPC L (140 kDa, Runs 23-27) after 2 min redispersion in deionized water in comparison to the particle sizes of the suspensions after milling and after $30 \mathrm{~h}$ storage (before redispersion).

Fig. 8. Particle sizes of the composites prepared using various precursor suspensions with (a) HPC SSL (Runs 3-7) and (b) HPC L (Runs 8-12) after 2 min redispersion in $0.05 \%$ SDS solution in comparison to the particle sizes of the suspensions after milling and after $30 \mathrm{~h}$ storage (before redispersion). Run 1 had no stabilizers.

Fig. 9. Drug dissolution profiles of the composites prepared using various precursor suspensions containing HPC with different molecular weights: (a) $40 \mathrm{kDa}$ (SSL grade, Runs 3-7) and (b) $140 \mathrm{kDa}$ (L grade, Runs 8-12). Run 1 had no stabilizers.

Fig. 10. Drug dissolution profiles of the composites prepared using various precursor suspensions with $0.05 \%$ SDS and HPC with different molecular weights: (a) $40 \mathrm{kDa}$ (SSL grade, Runs 13-17), (b) 100 kDa (SL grade, Runs 18-22), and (c) 140 kDa (L grade, Runs 23-27). Run 2 had only $0.05 \%$ SDS. 


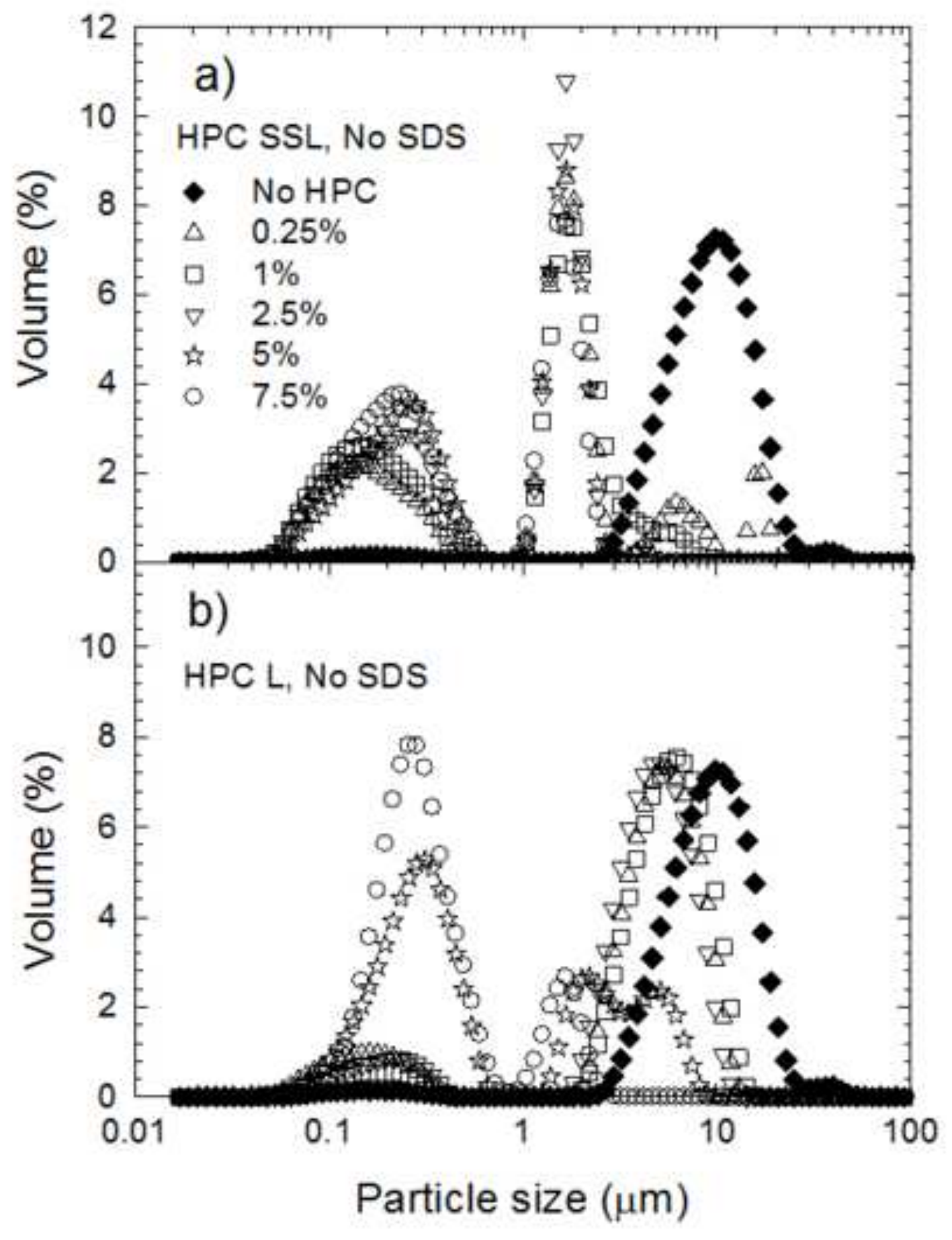




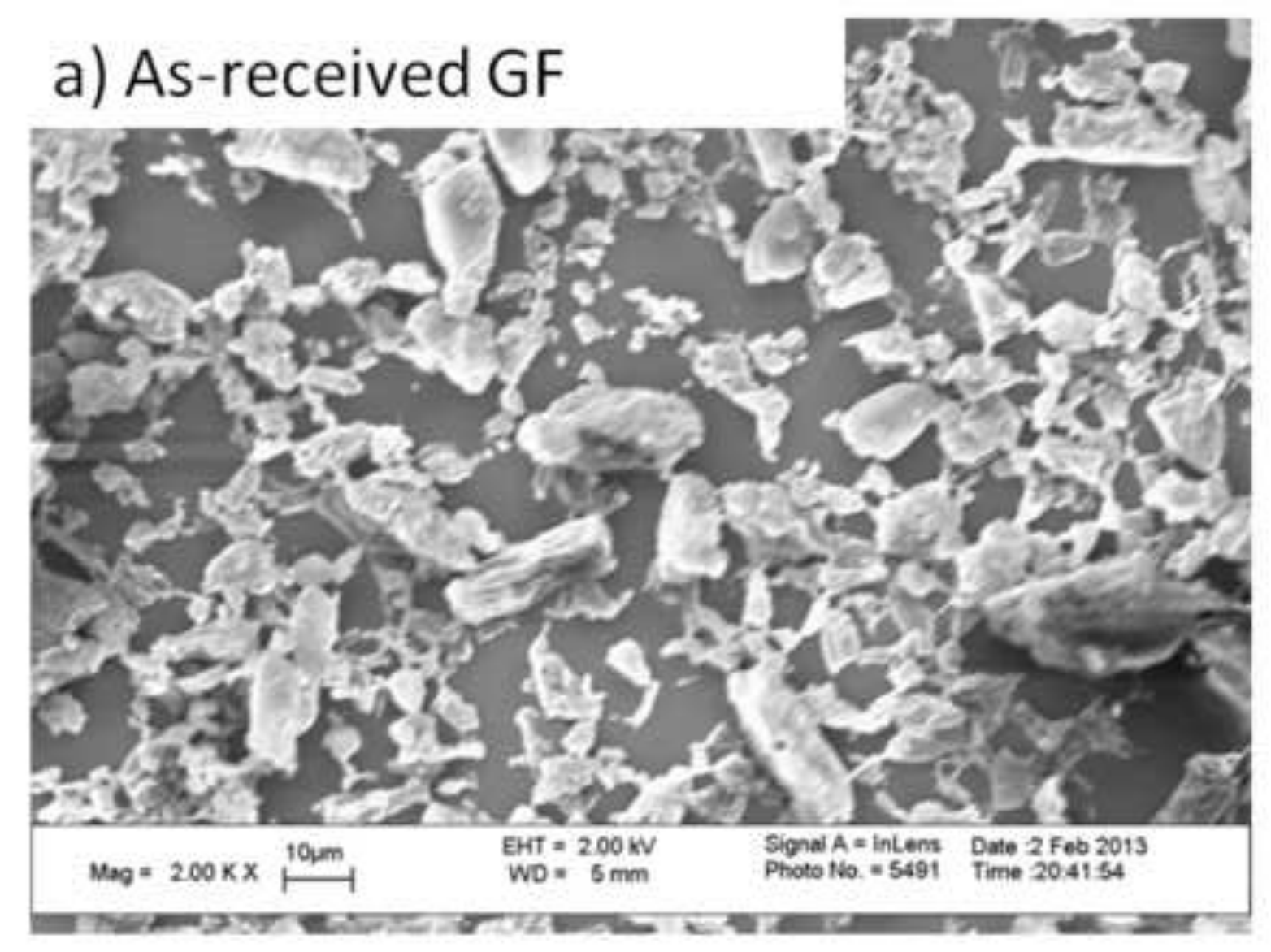




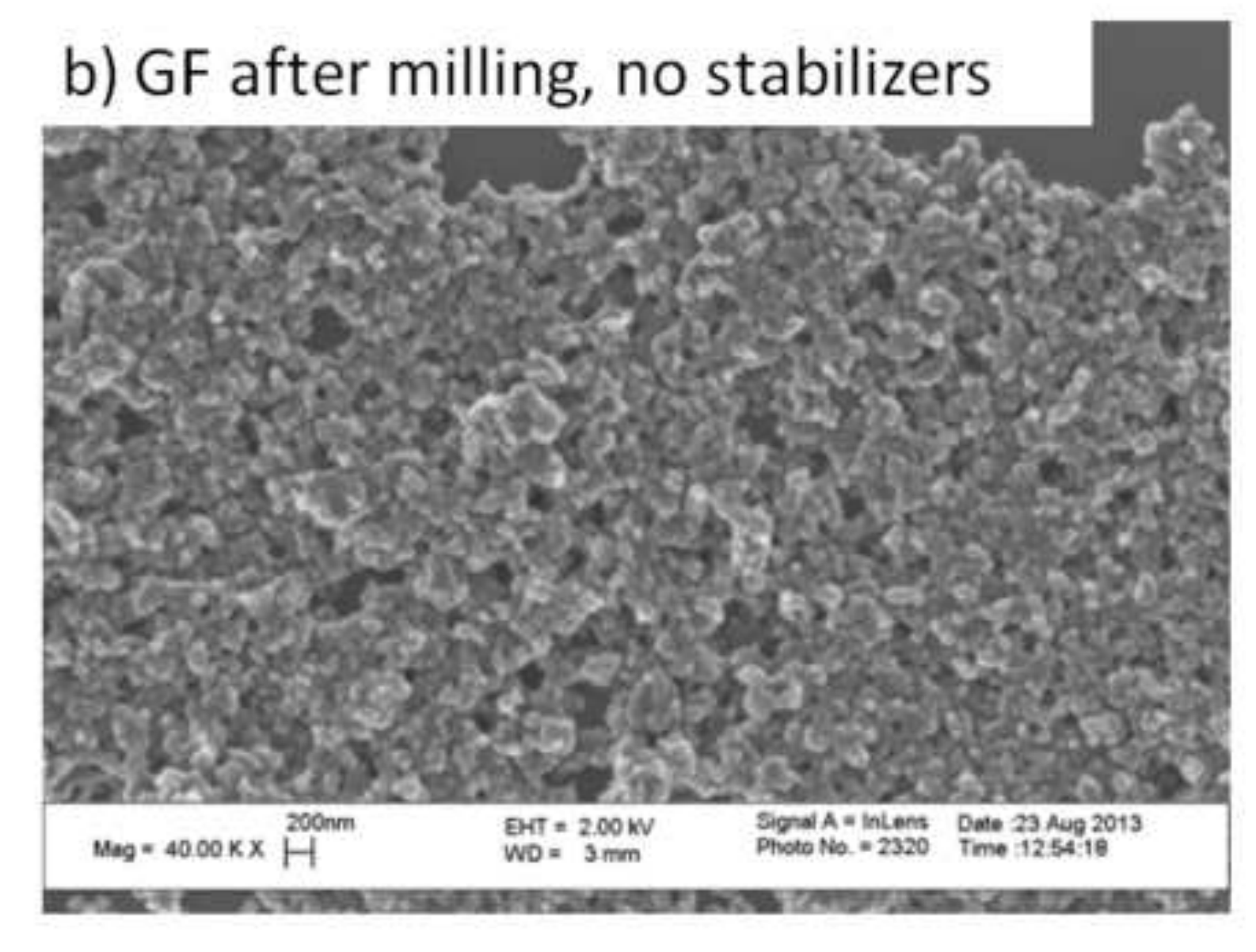

\section{.}

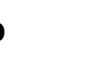




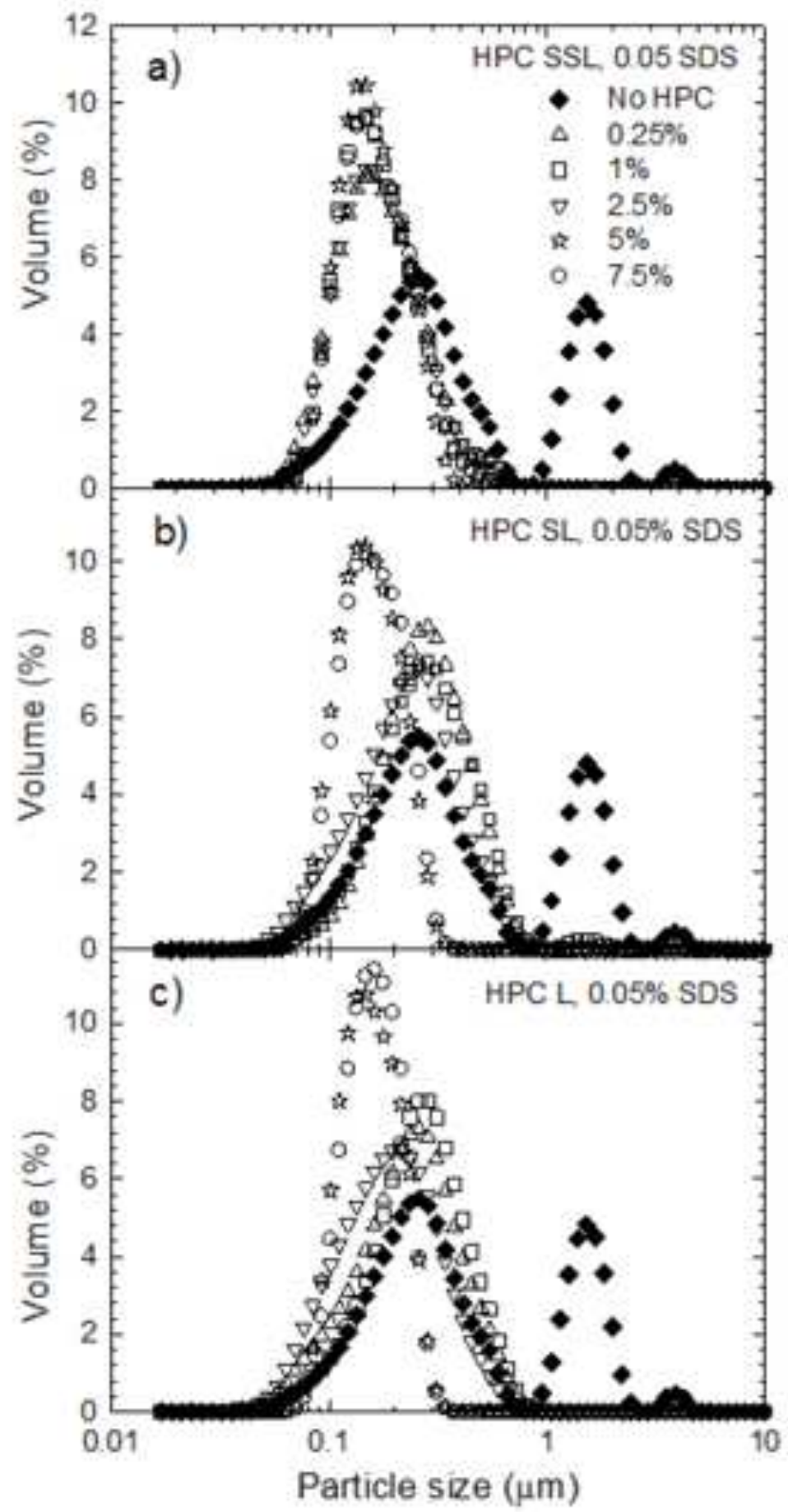




\section{b) $1 \% \mathrm{HPC} \mathrm{SSL}, 0.05 \% \mathrm{SDS}$}

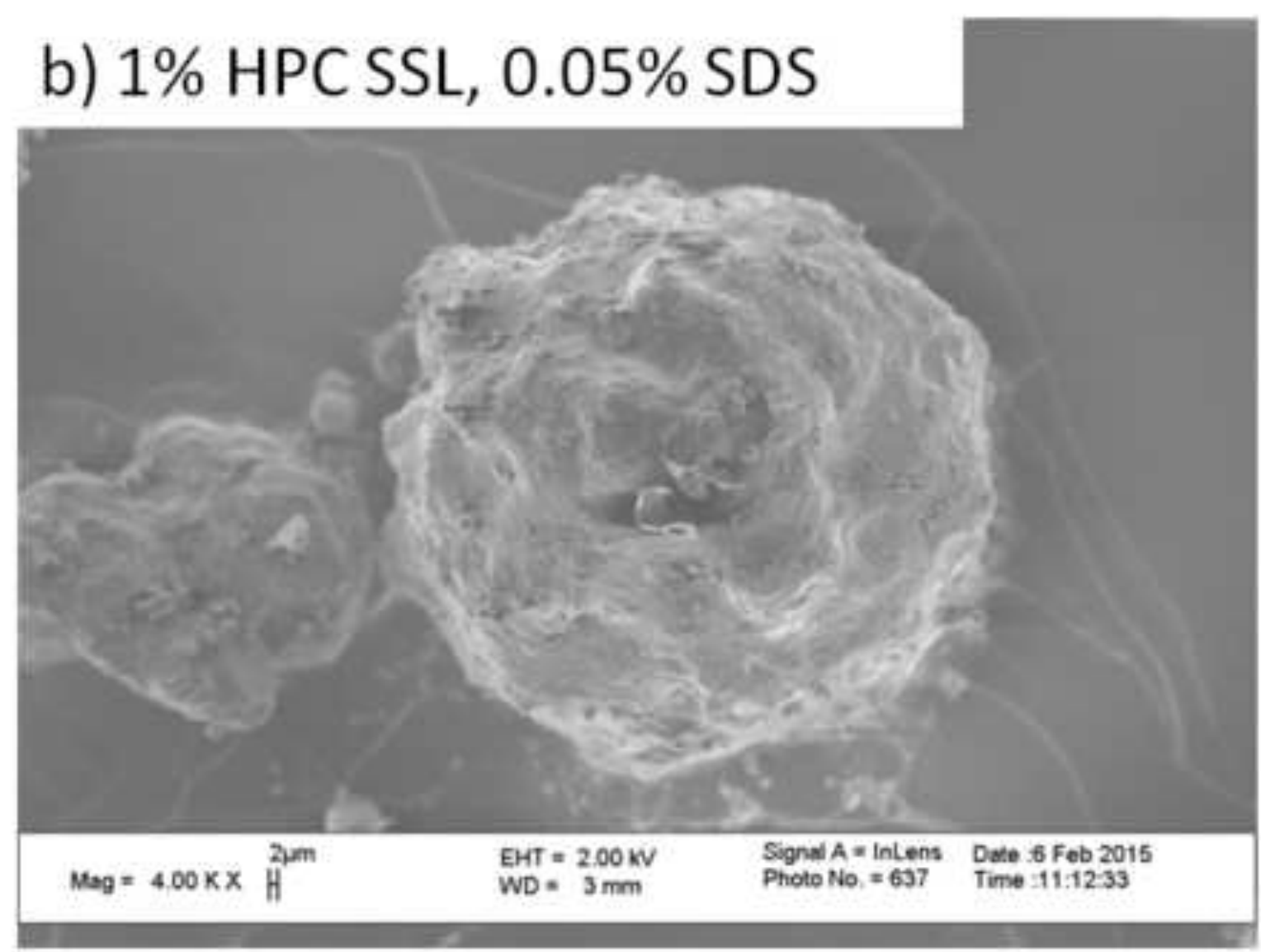


c) $1 \% \mathrm{HPC}$ SSL, $0.05 \% \mathrm{SDS}$

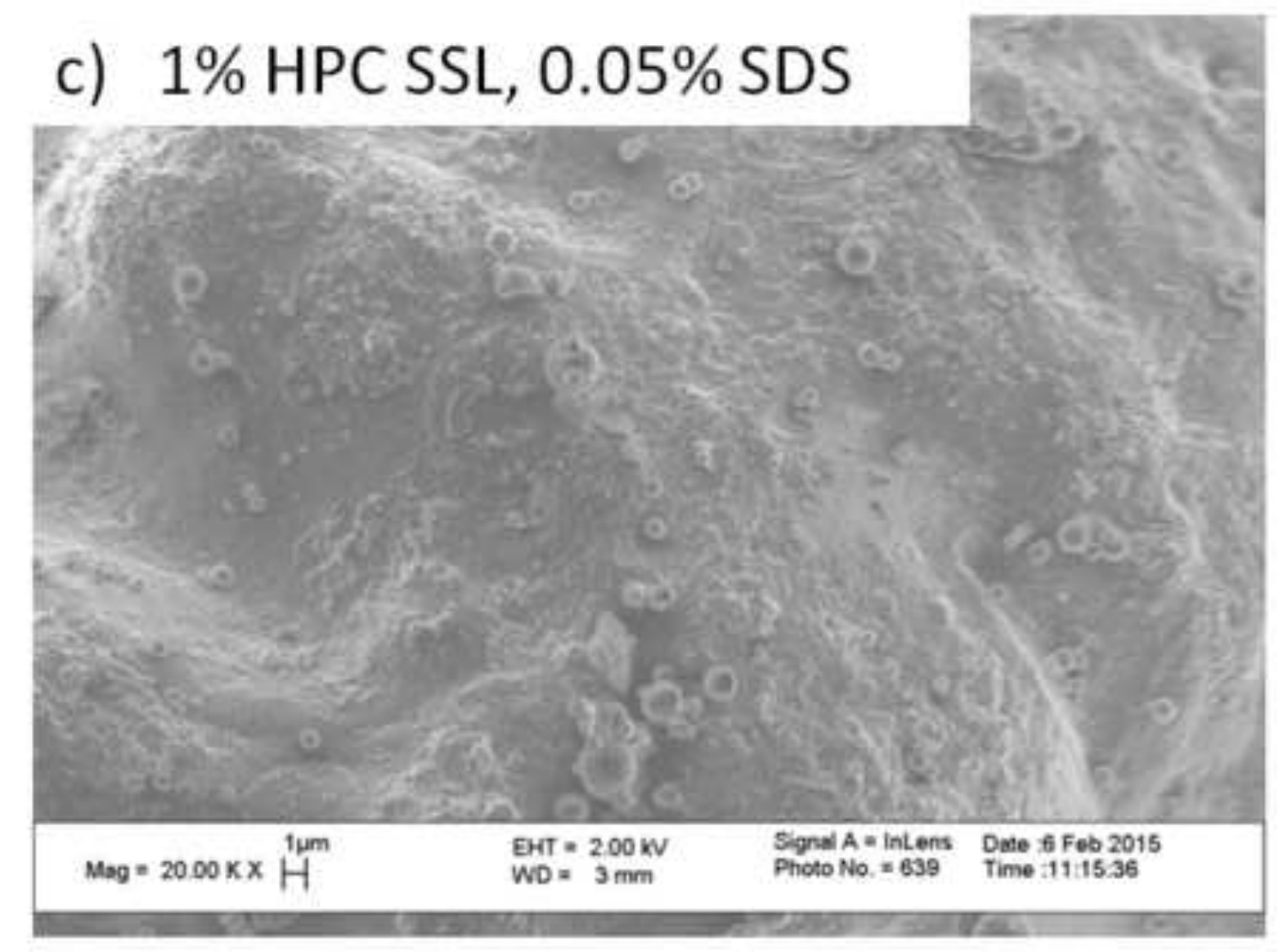




\section{Figure $4 d$}

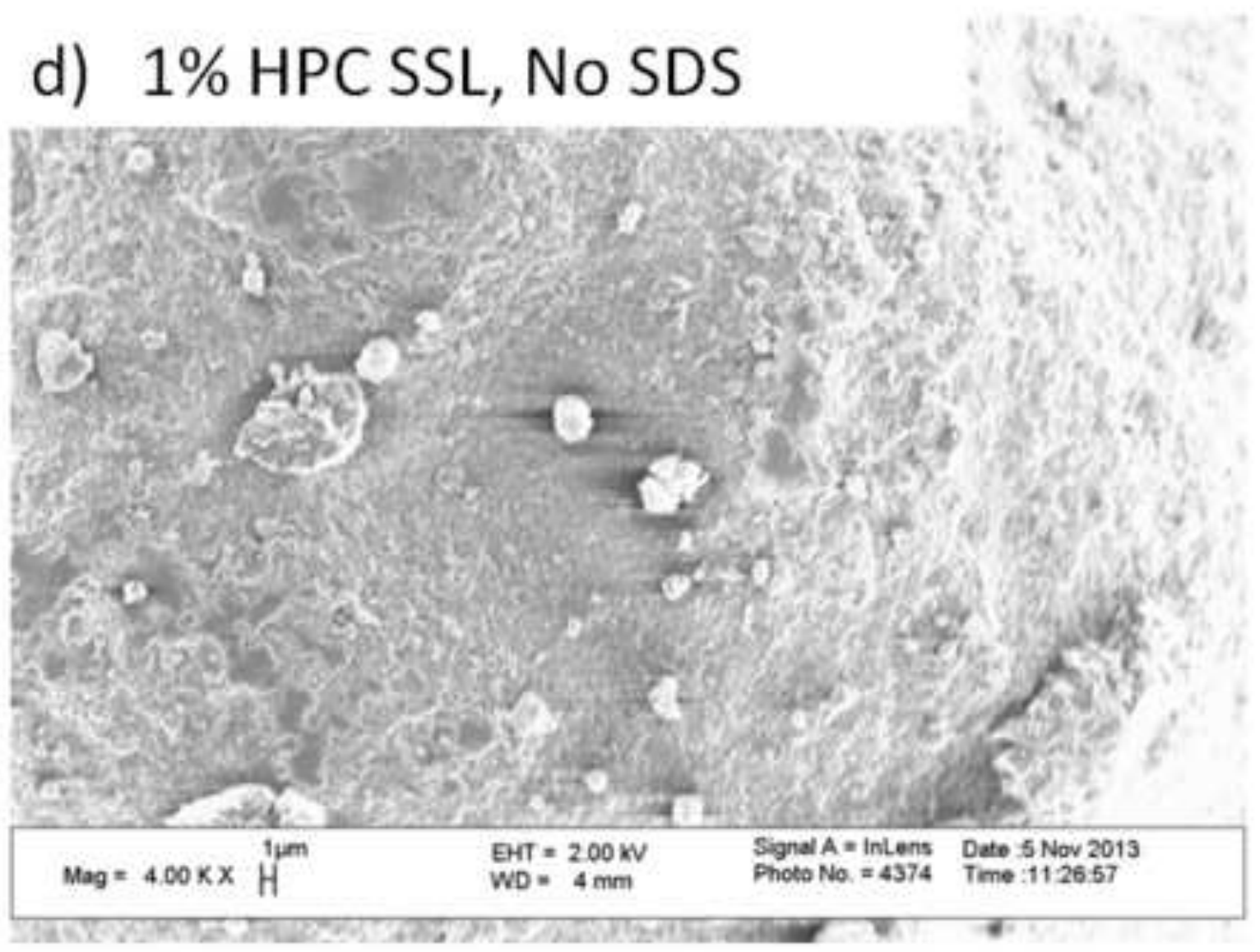




\section{e) $5 \% \mathrm{HPC}$ SSL, $0.05 \% \mathrm{SDS}$}

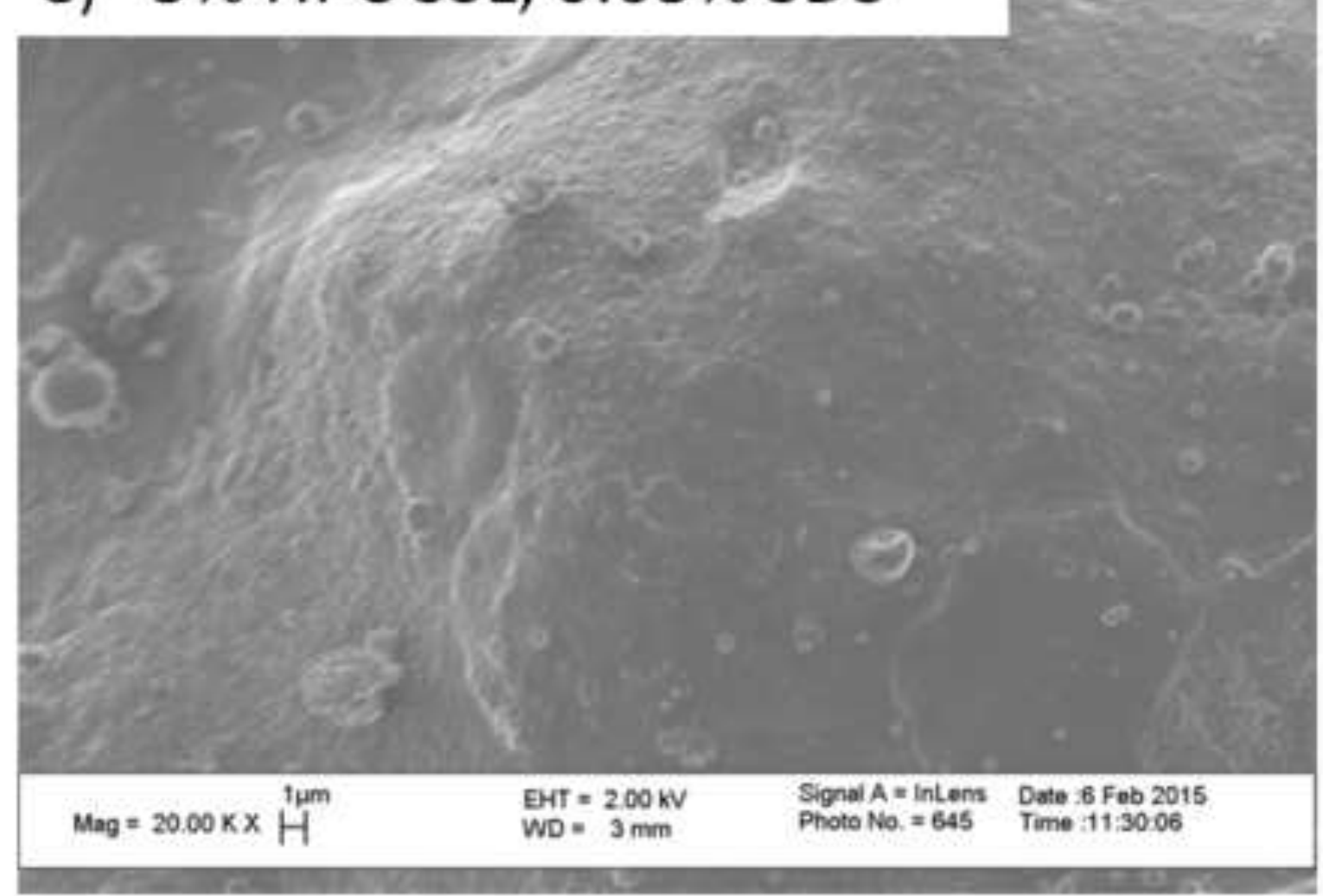


g) $5 \% \mathrm{HPC}$ L, $0.05 \% \mathrm{SDS}$

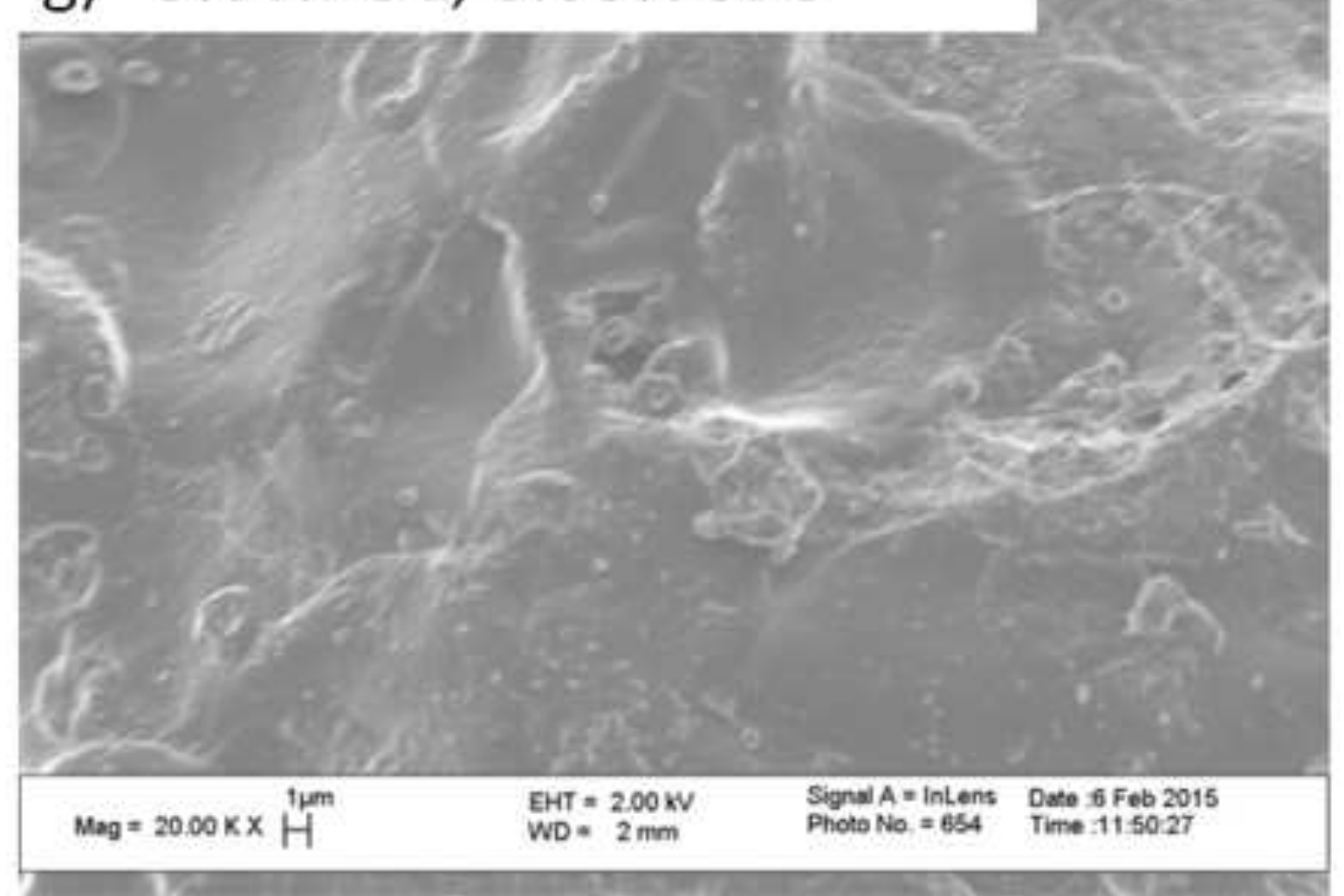




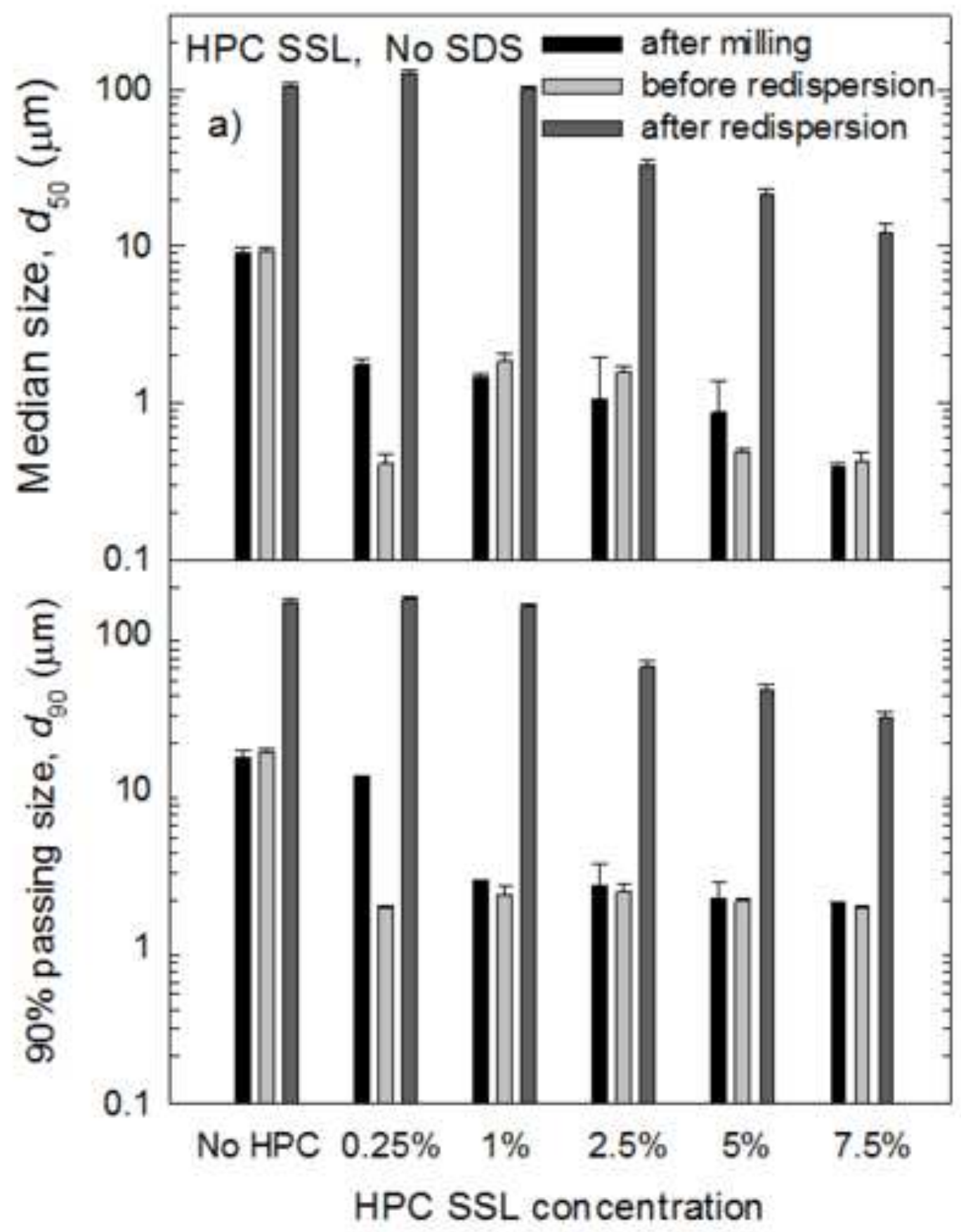




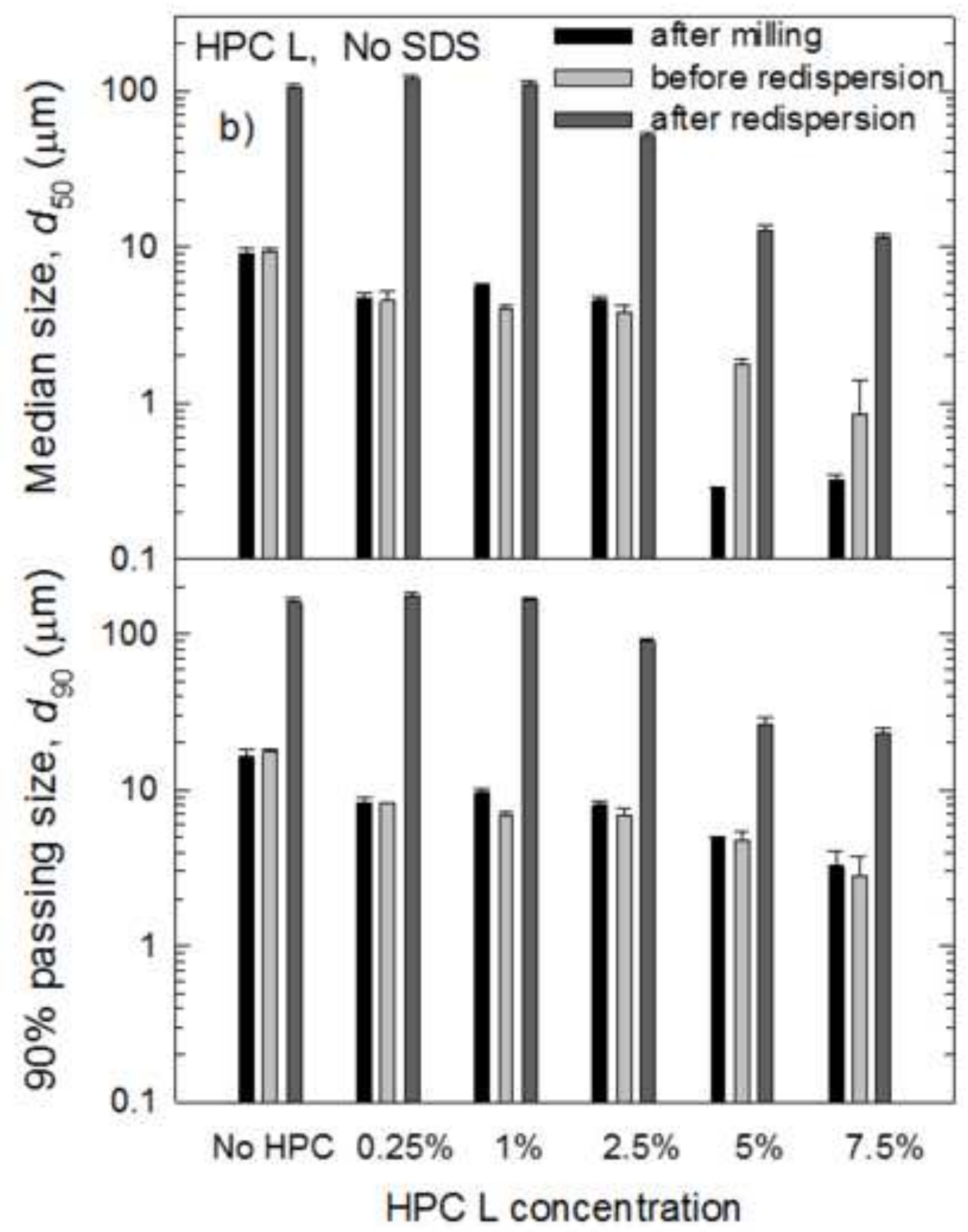




\section{Figure $6 a$

a) No HPC, No SDS

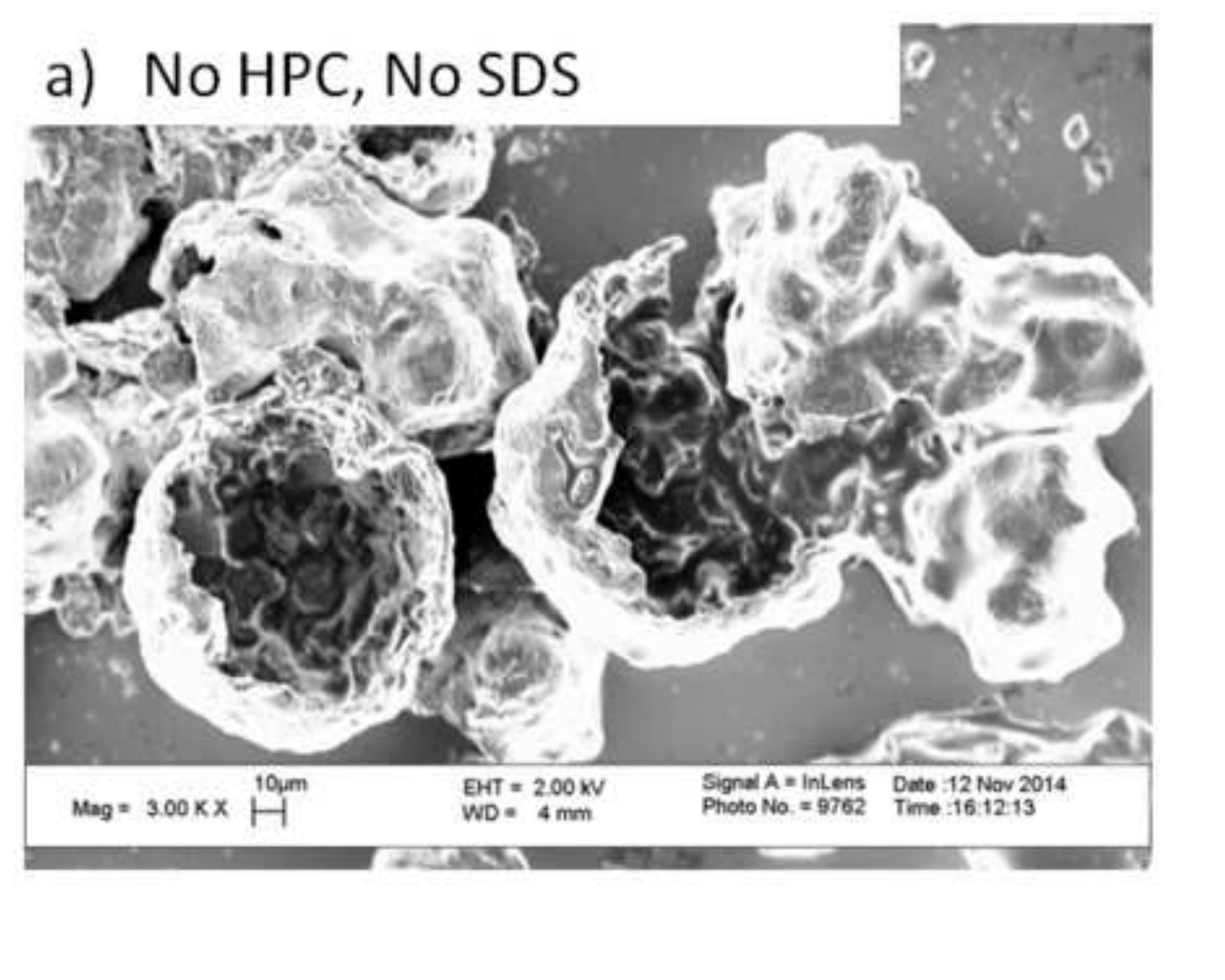

.

.

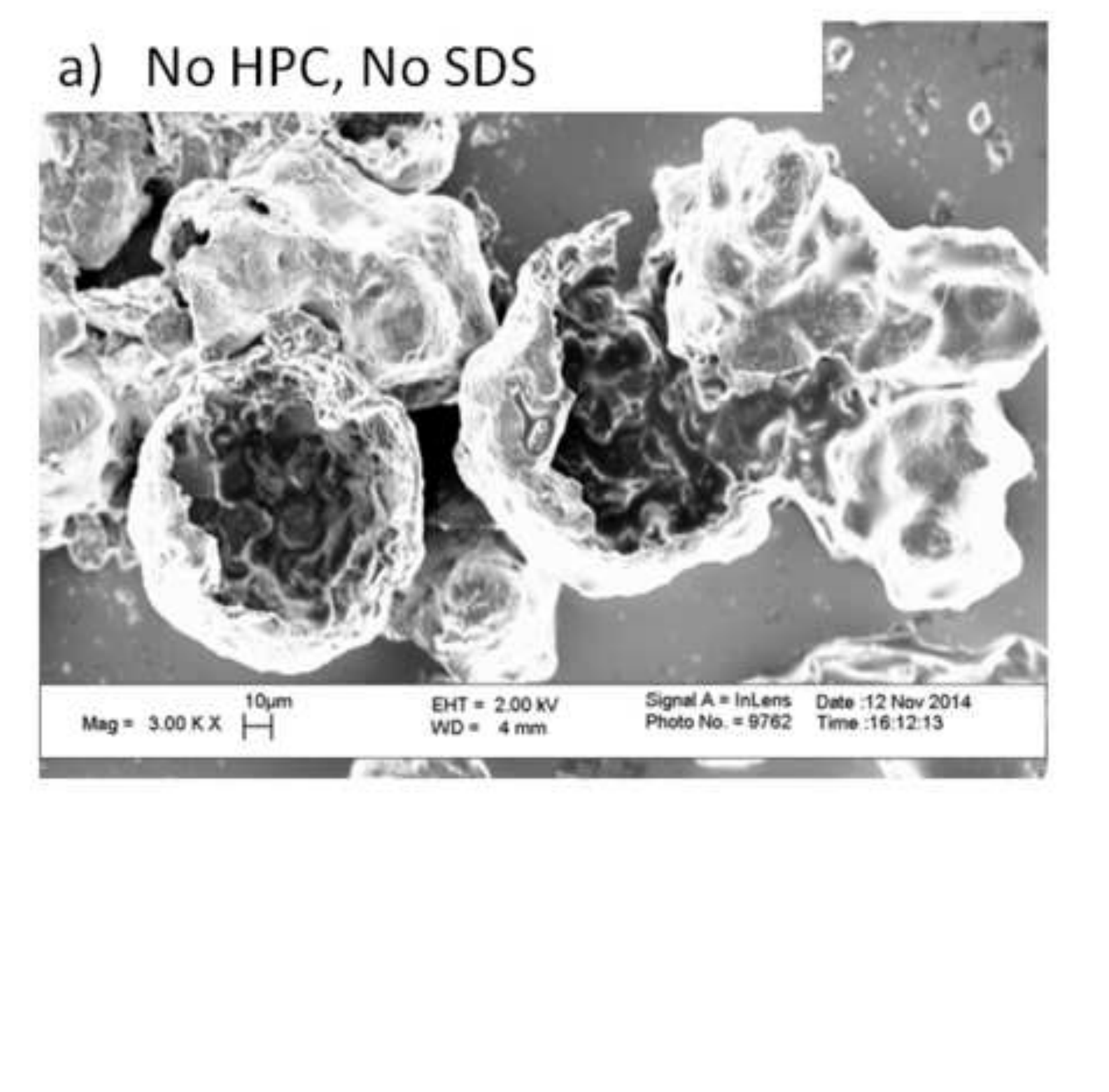

.
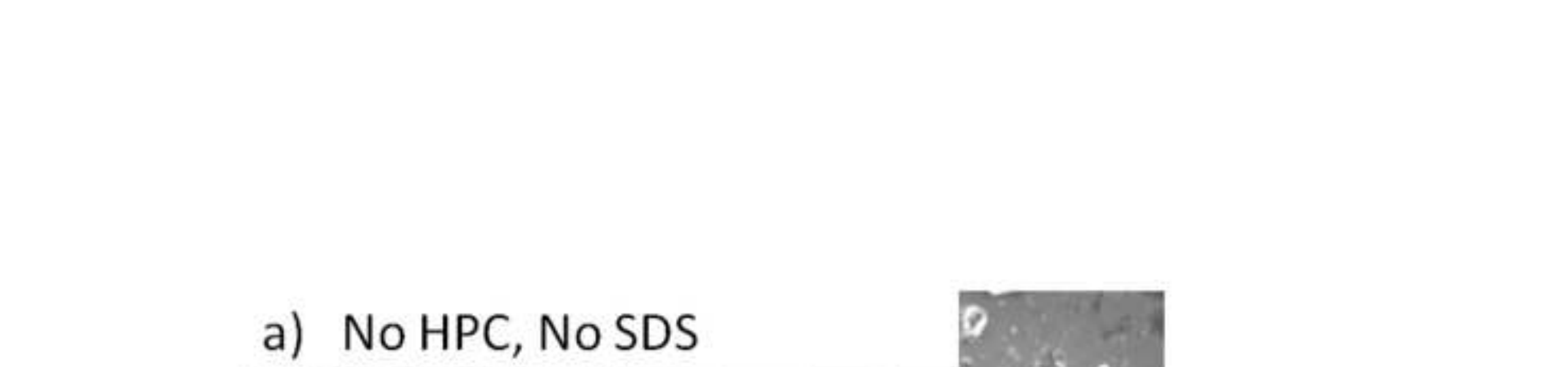

.
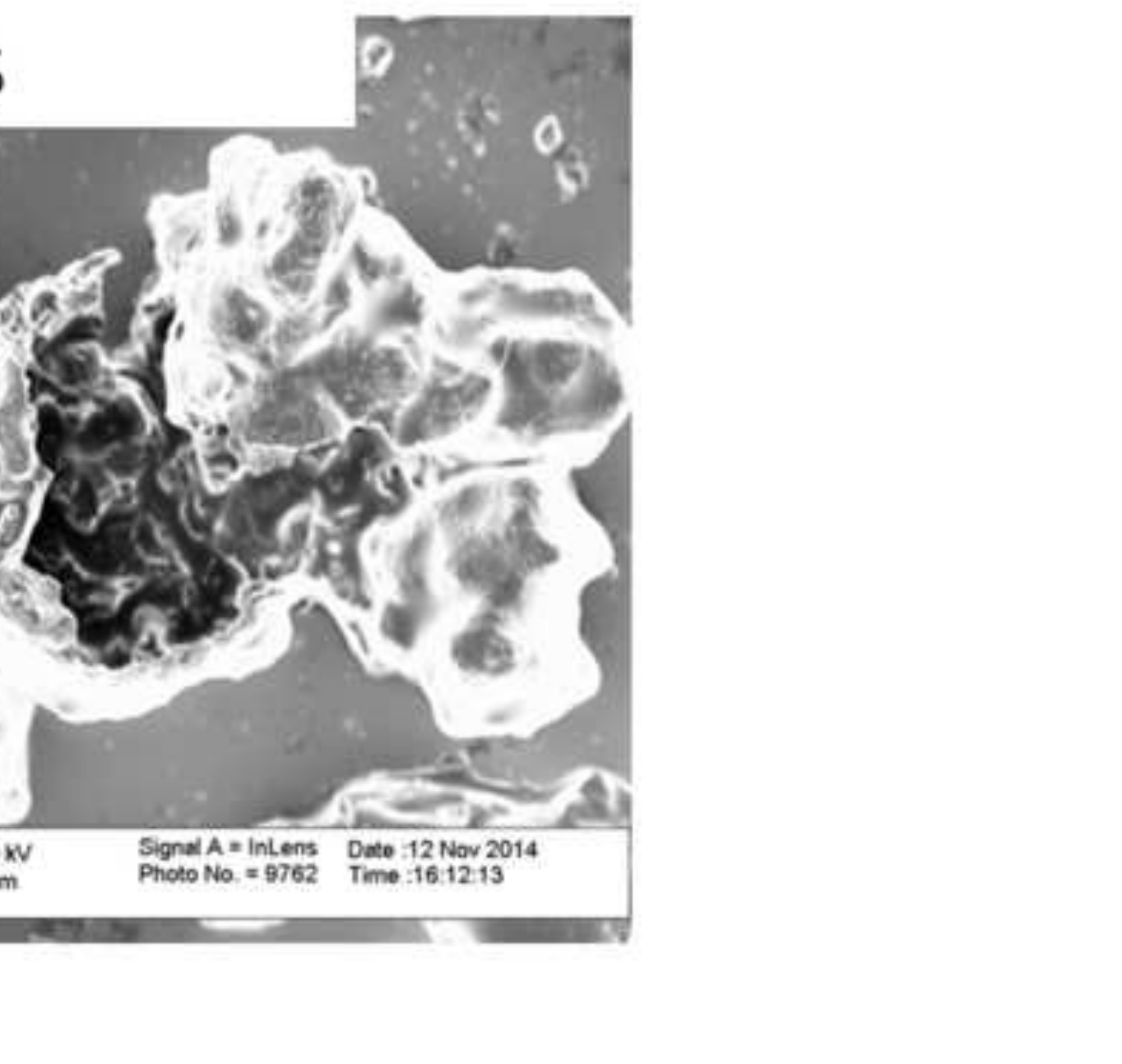


\section{b) $7.5 \% \mathrm{HPC} \mathrm{SSL}$}

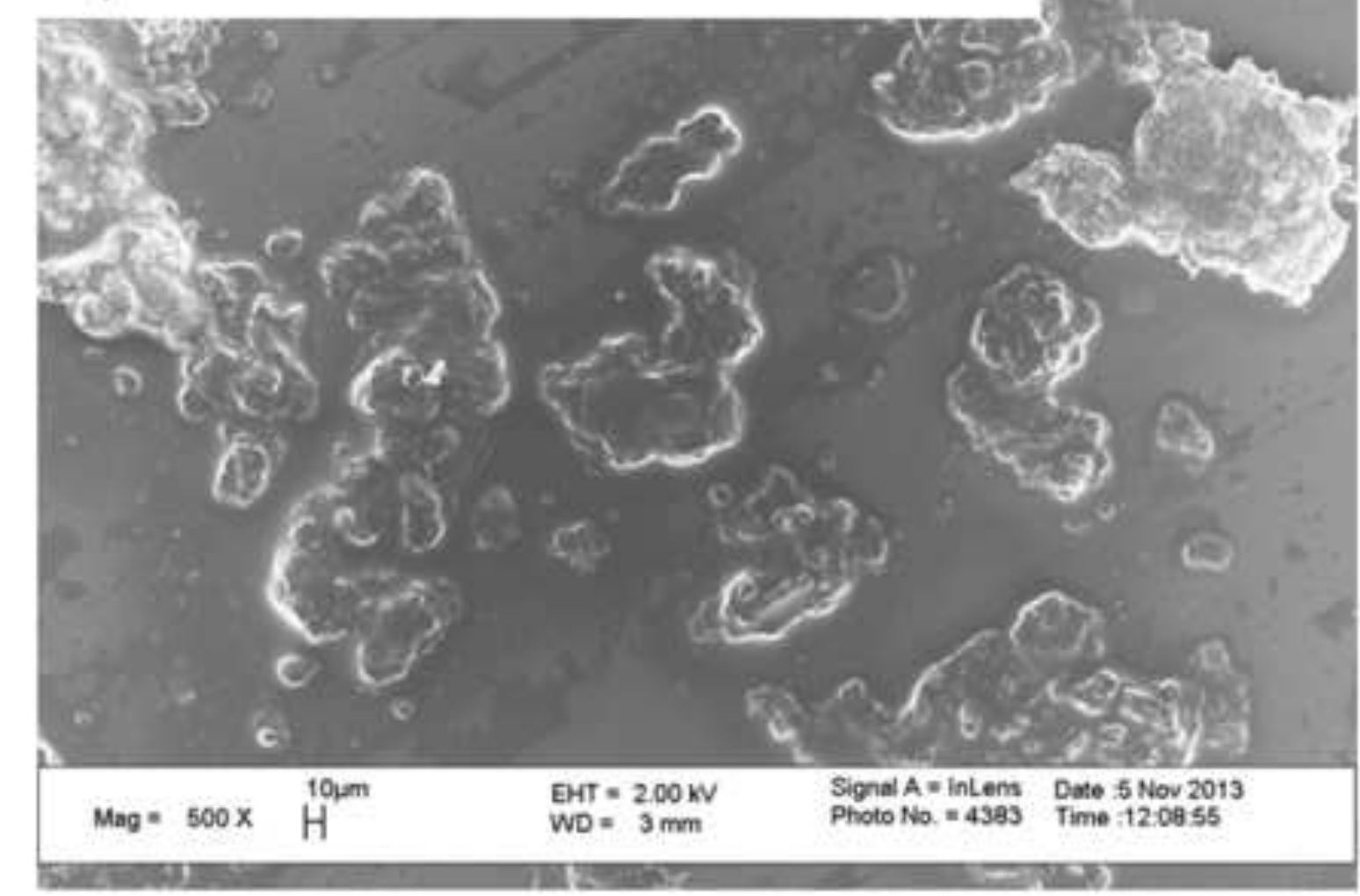

$=3 \times 1$ 


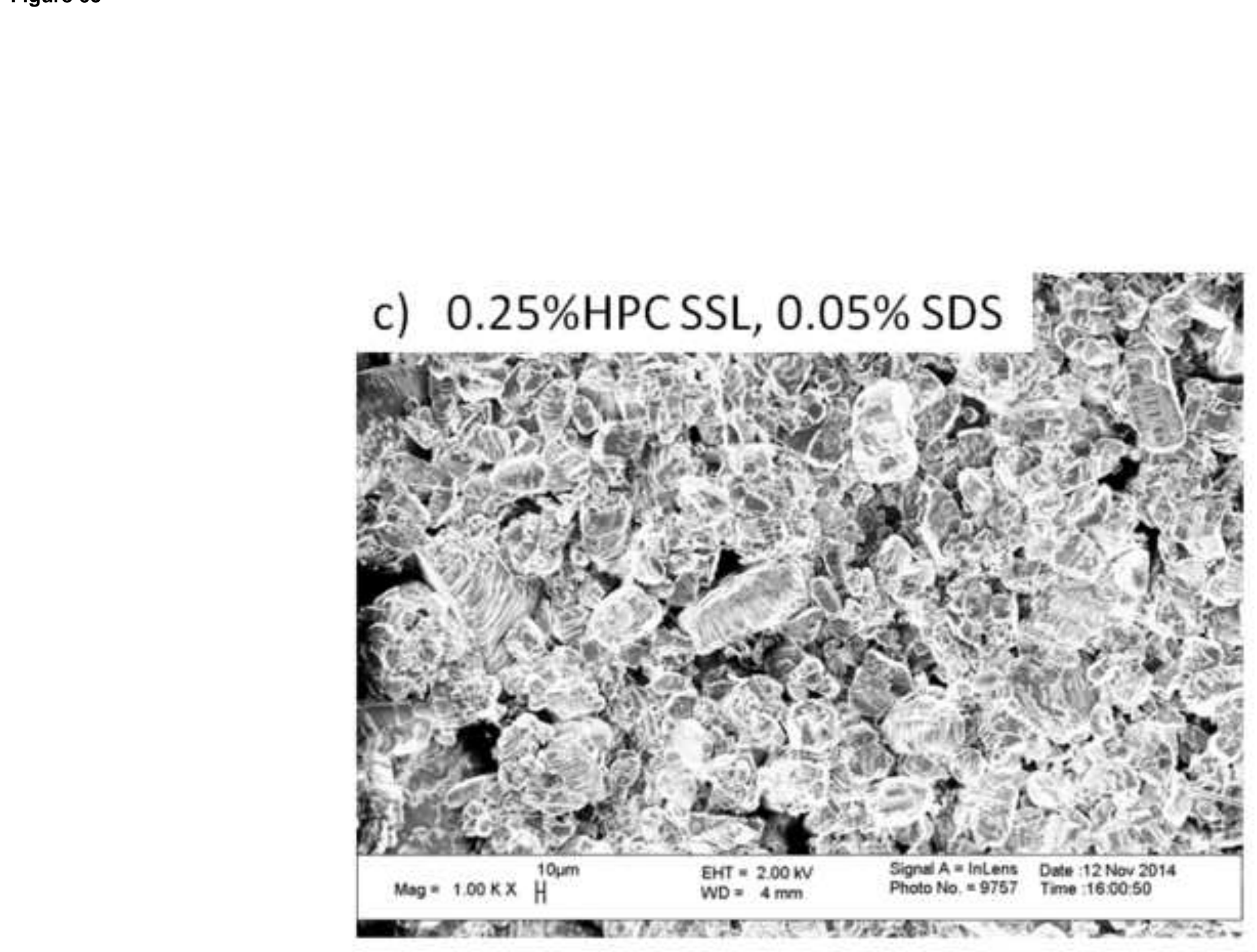

.
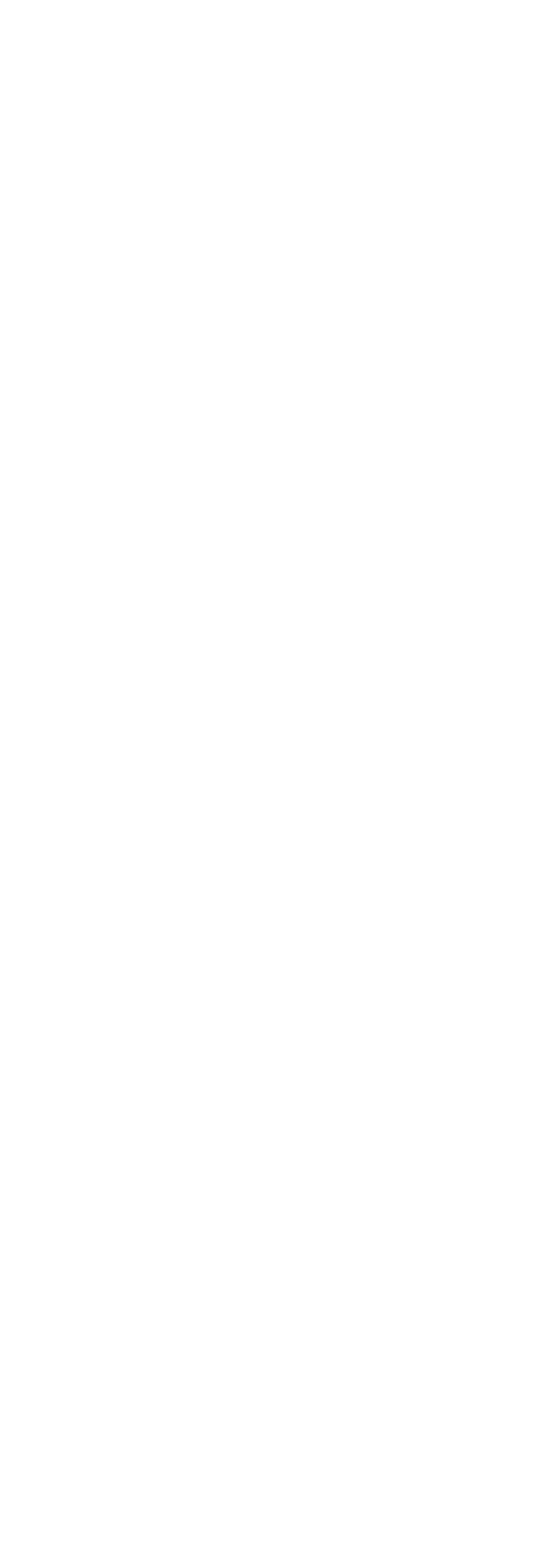


\section{Figure $6 \mathrm{~d}$}

\section{d) $5 \% \mathrm{HPC} \mathrm{SSL}, 0.05 \% \mathrm{SDS}$}
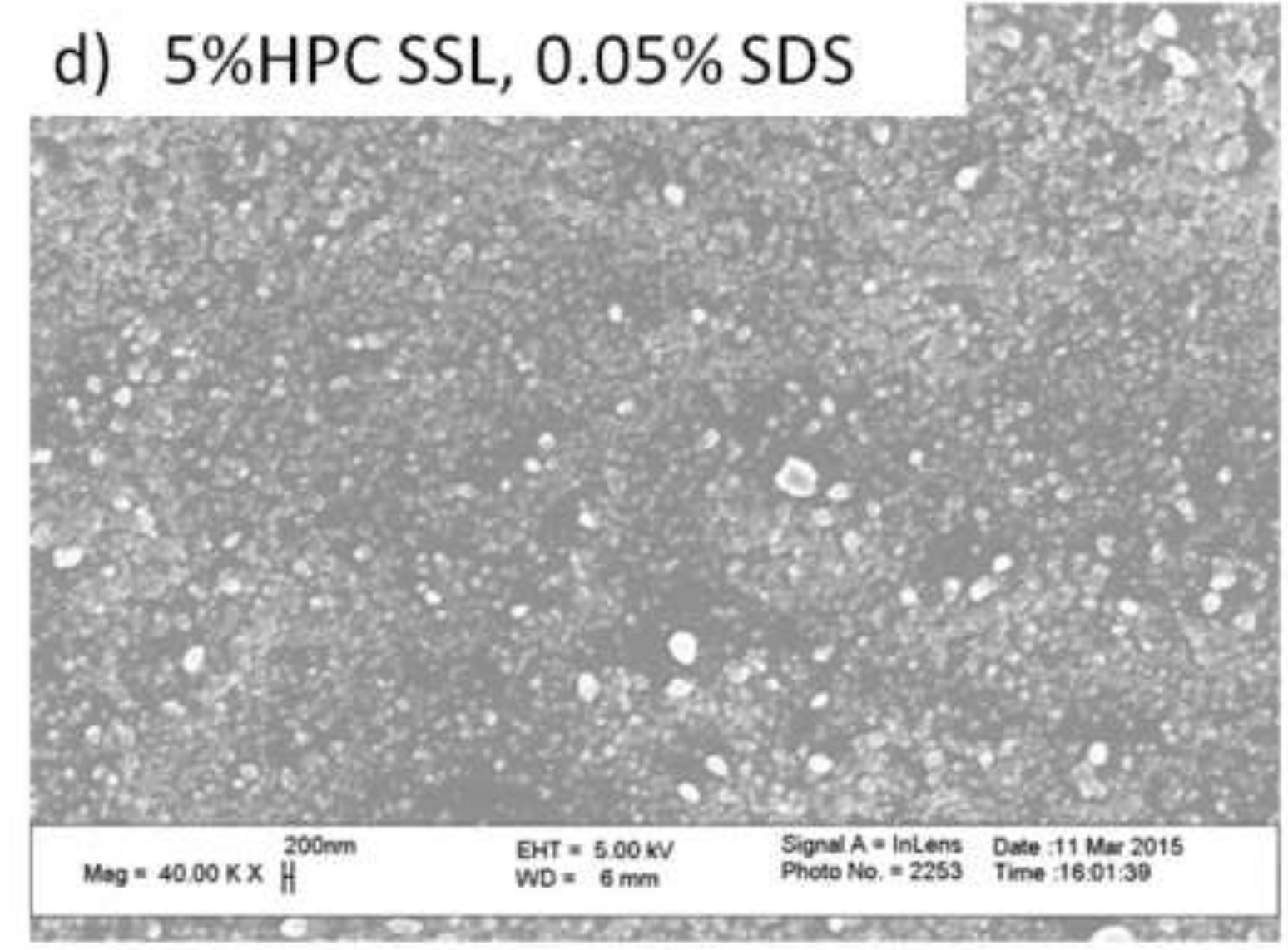


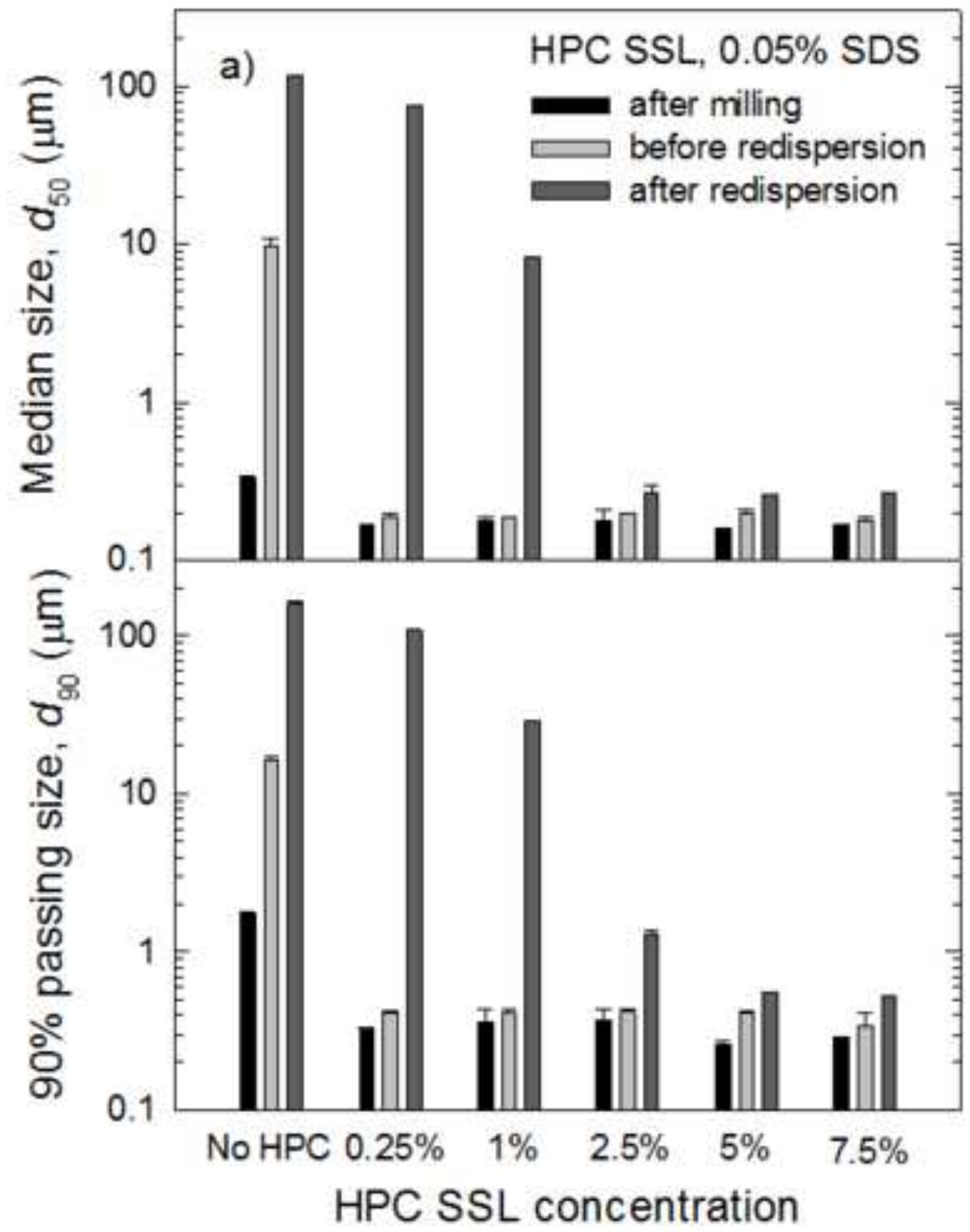




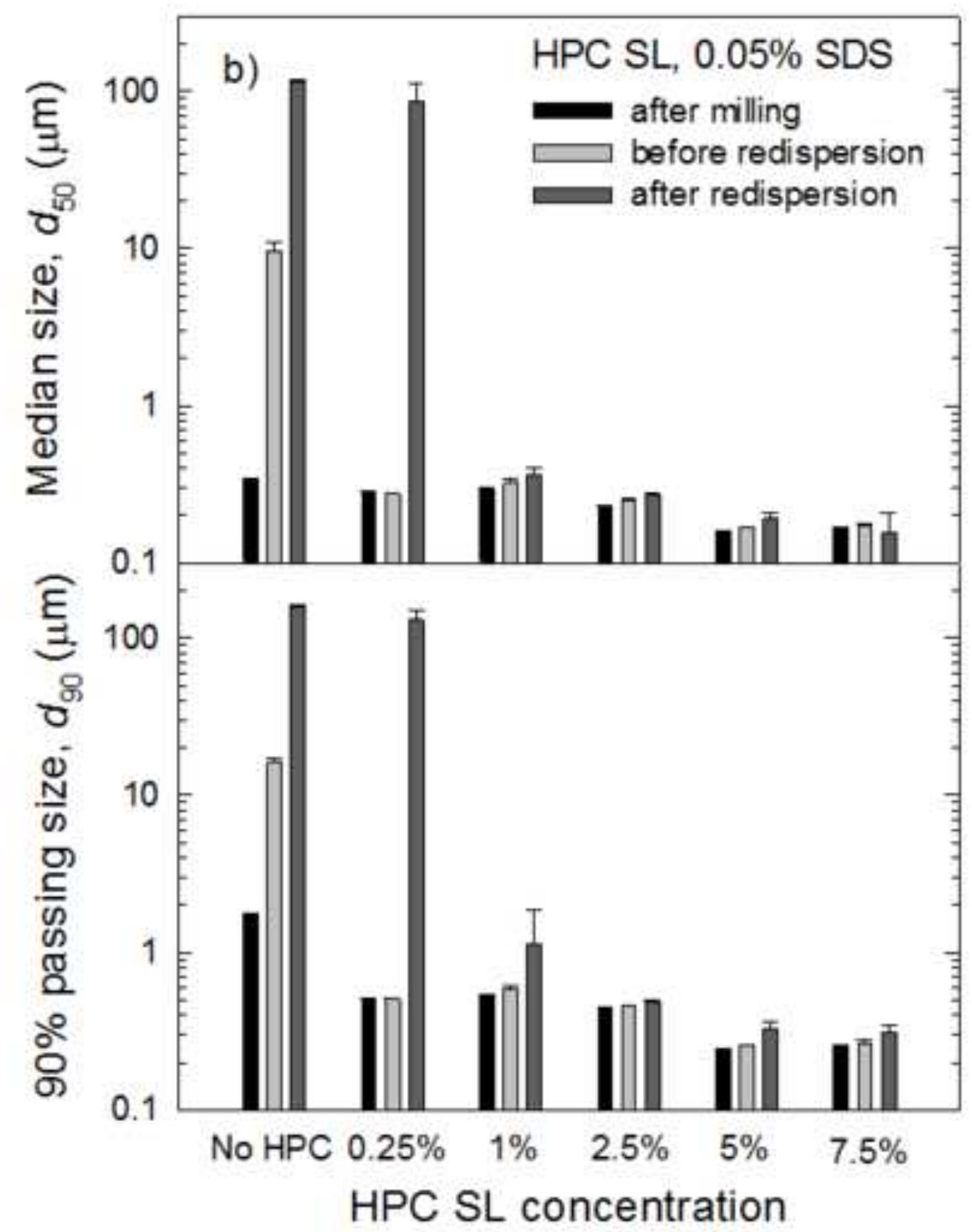




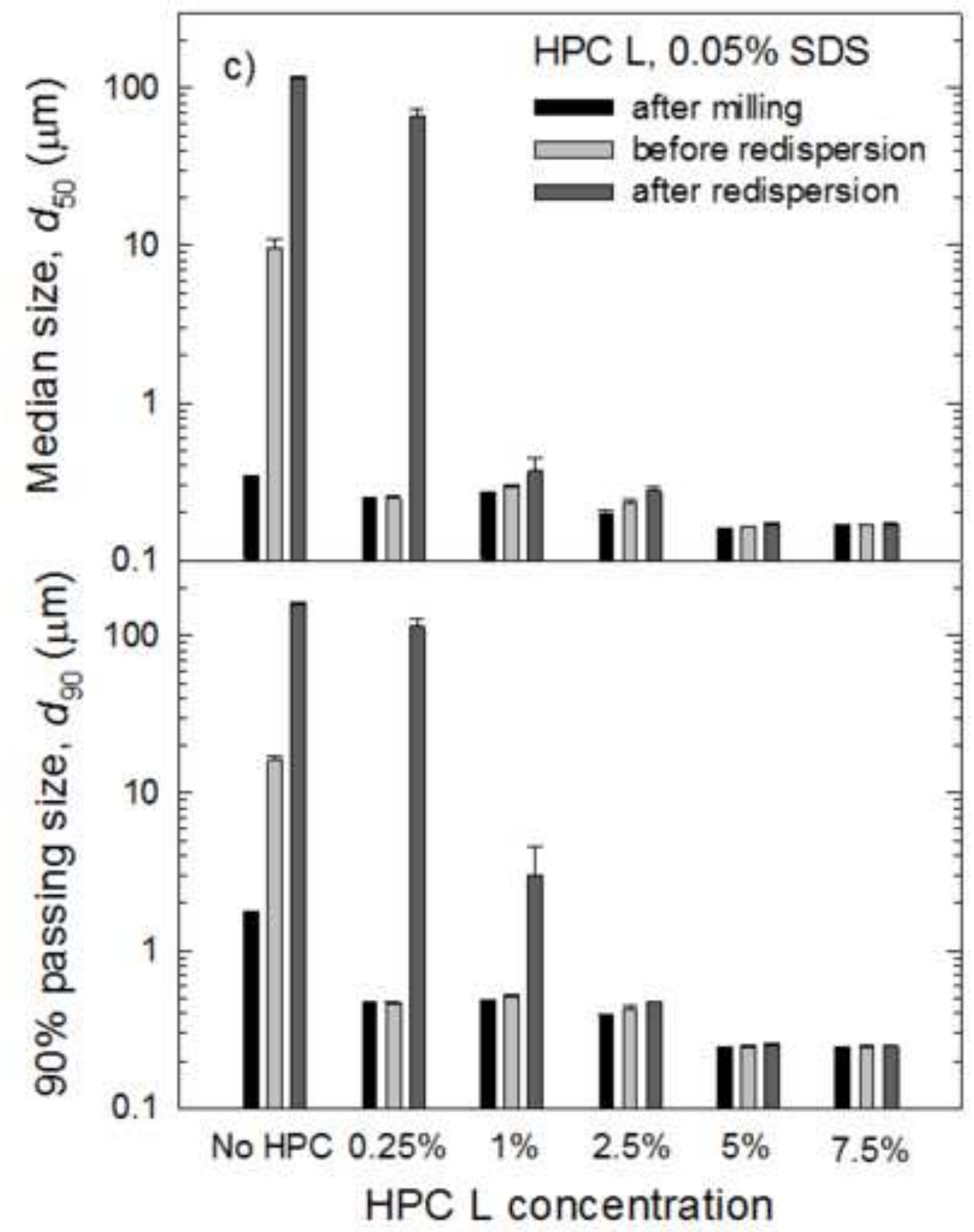









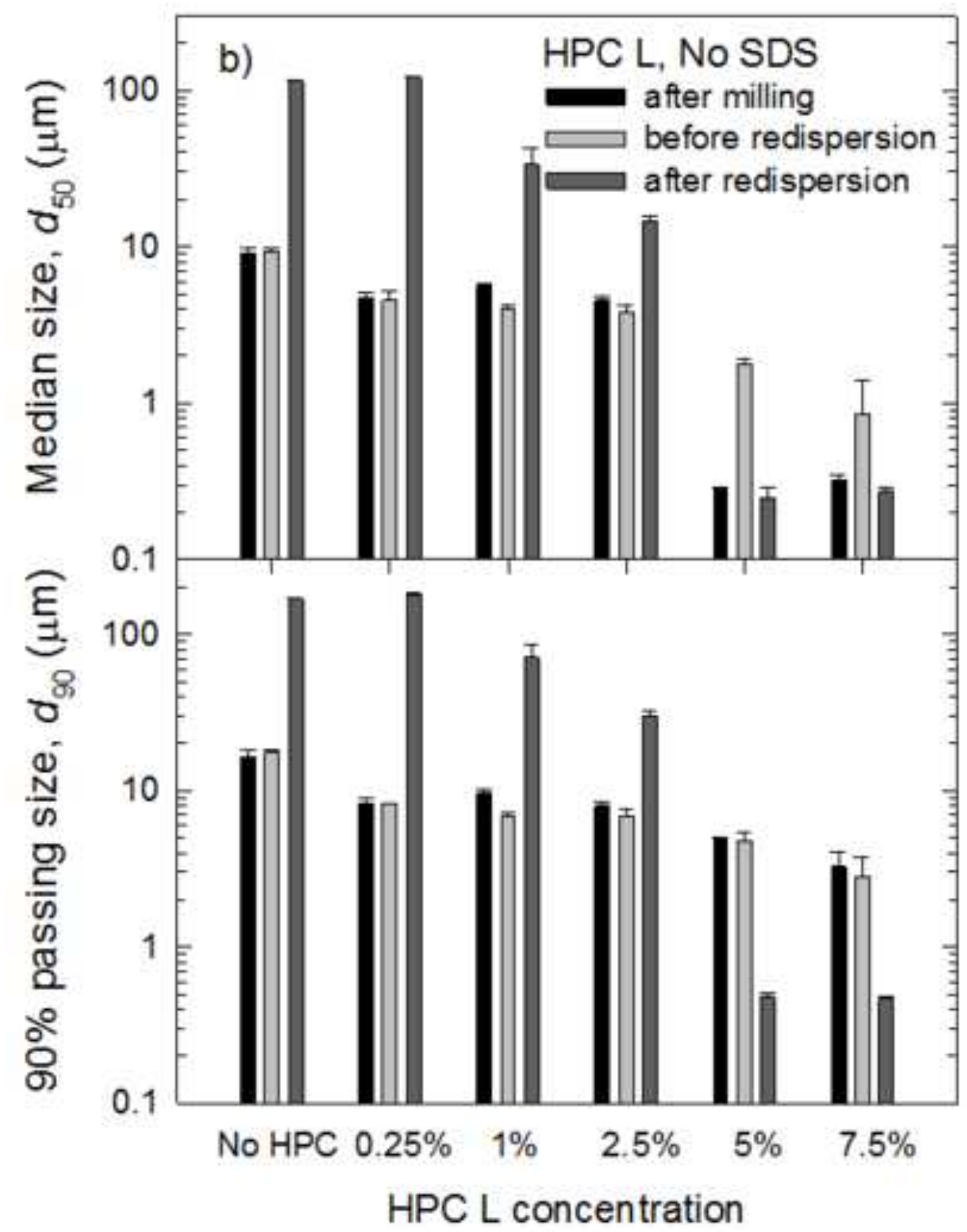




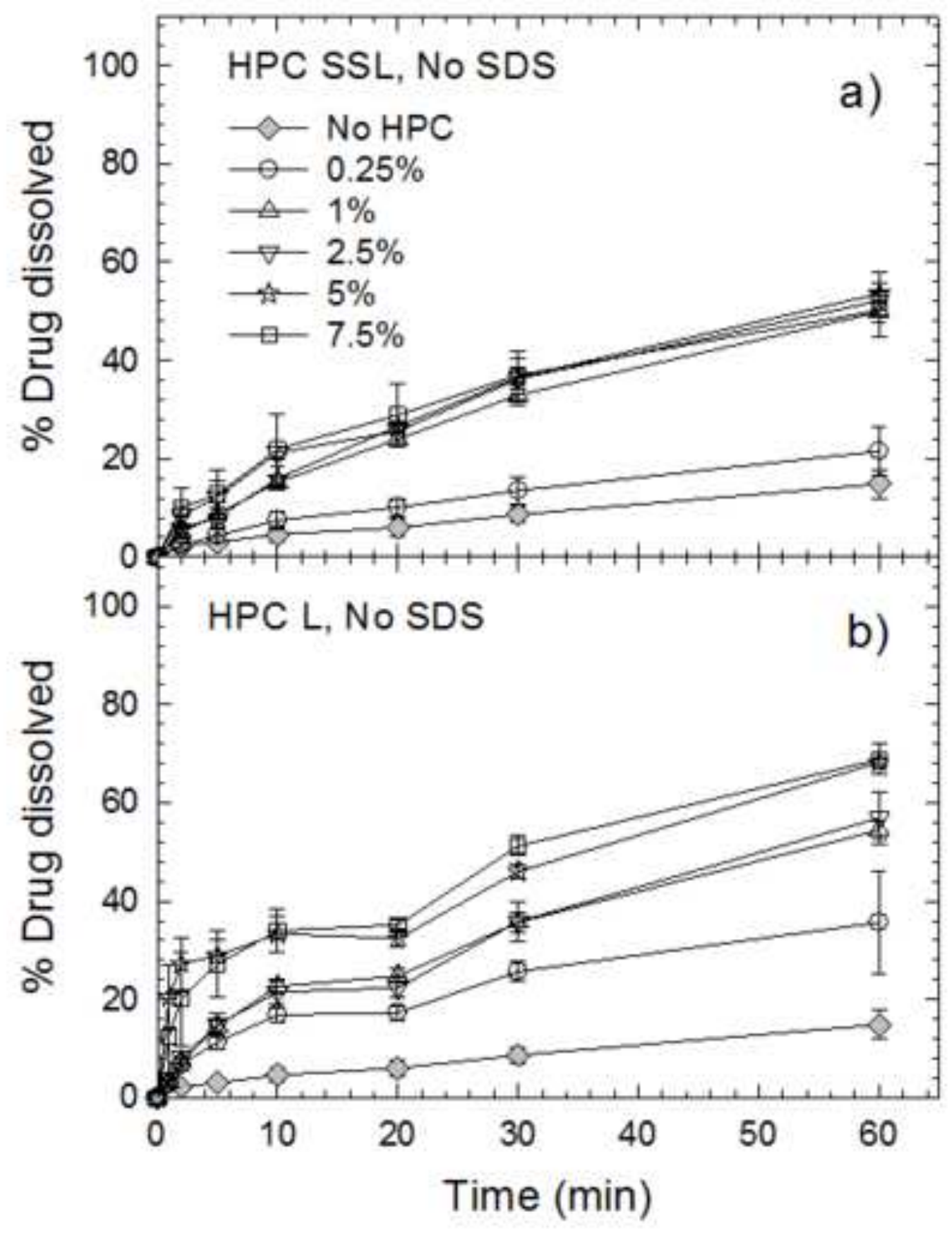




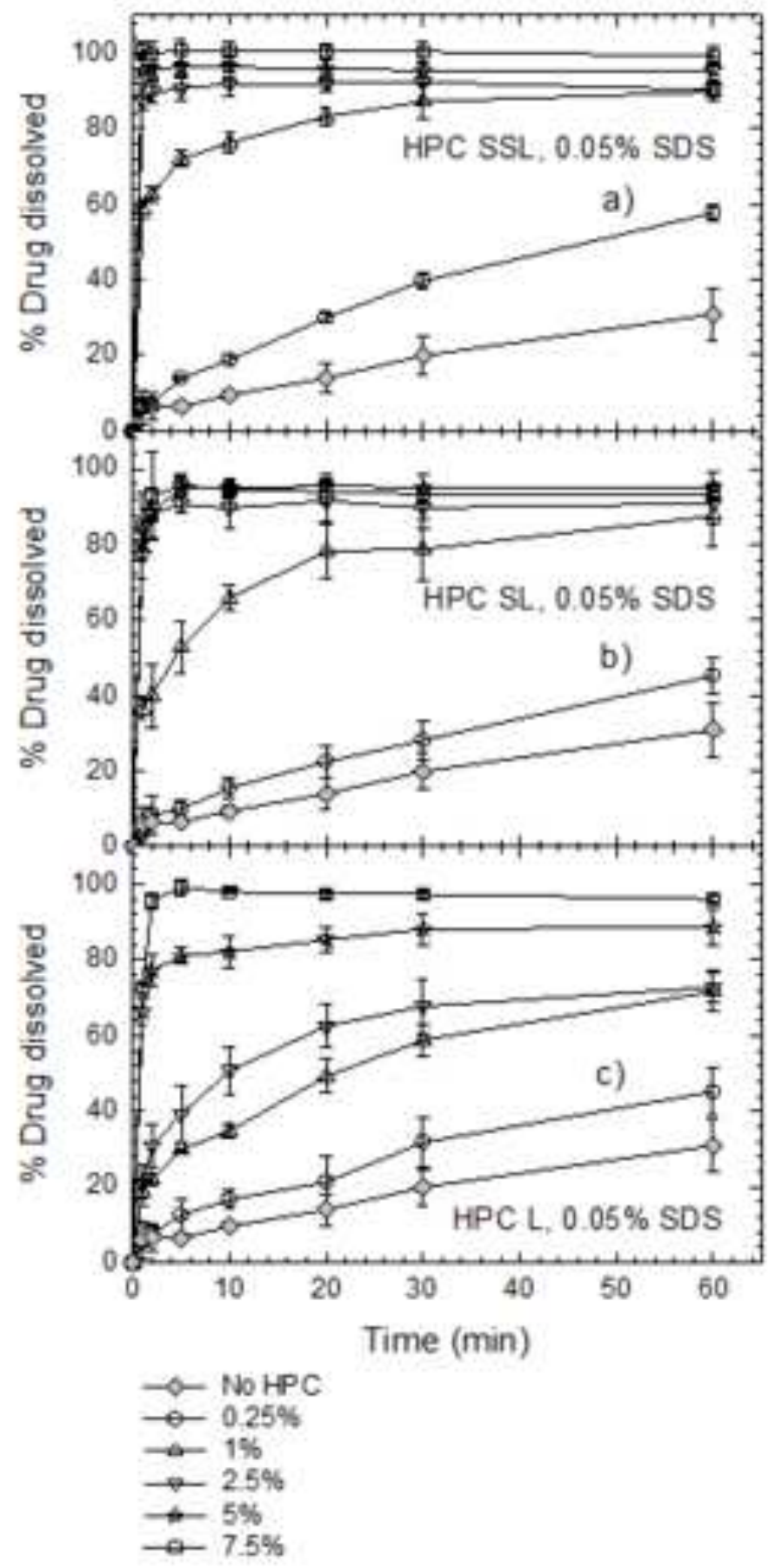




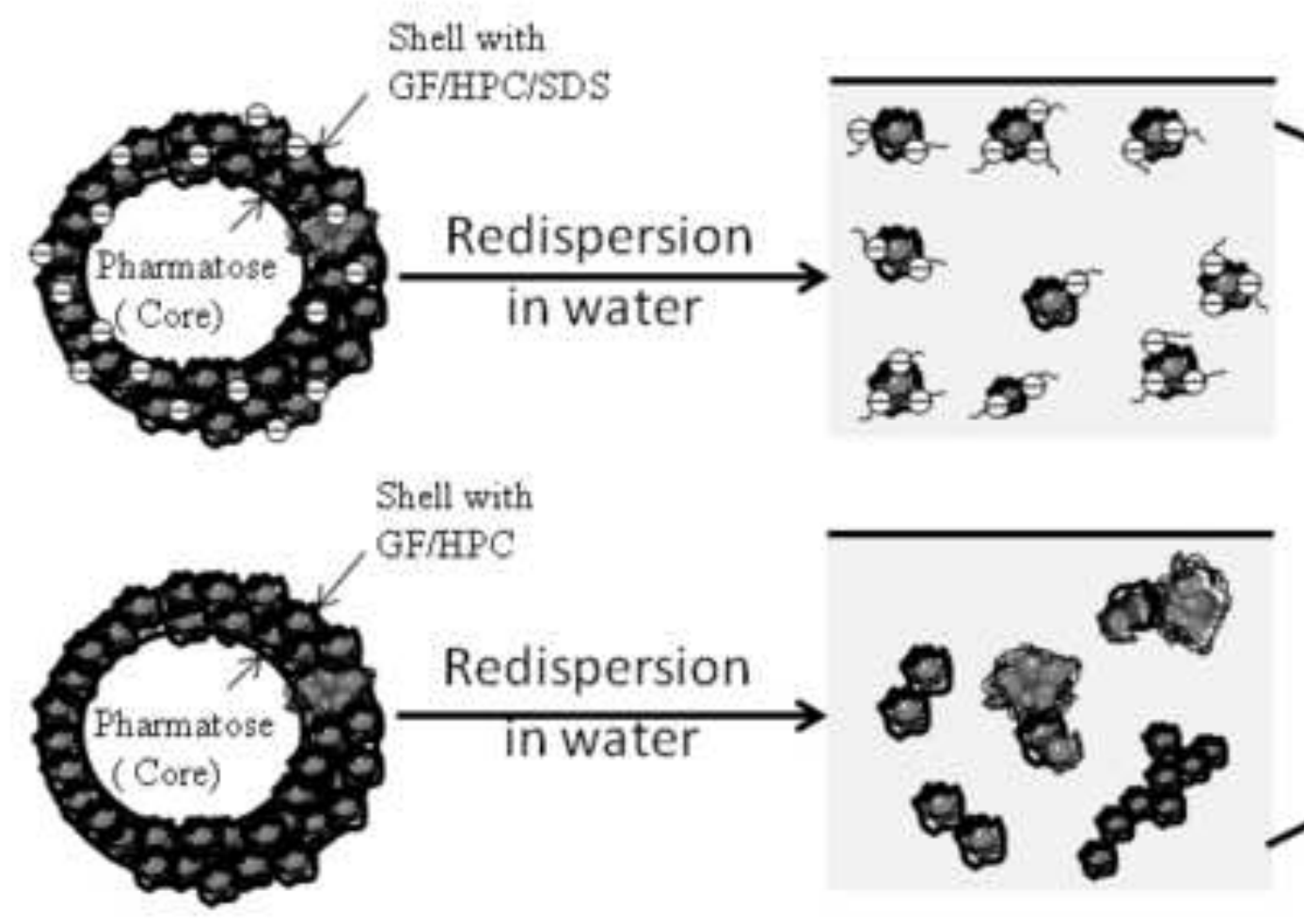

Fluidized bed dried drug composite particles
Drug (GF) nanoparticles/clusters recovered from redispersion

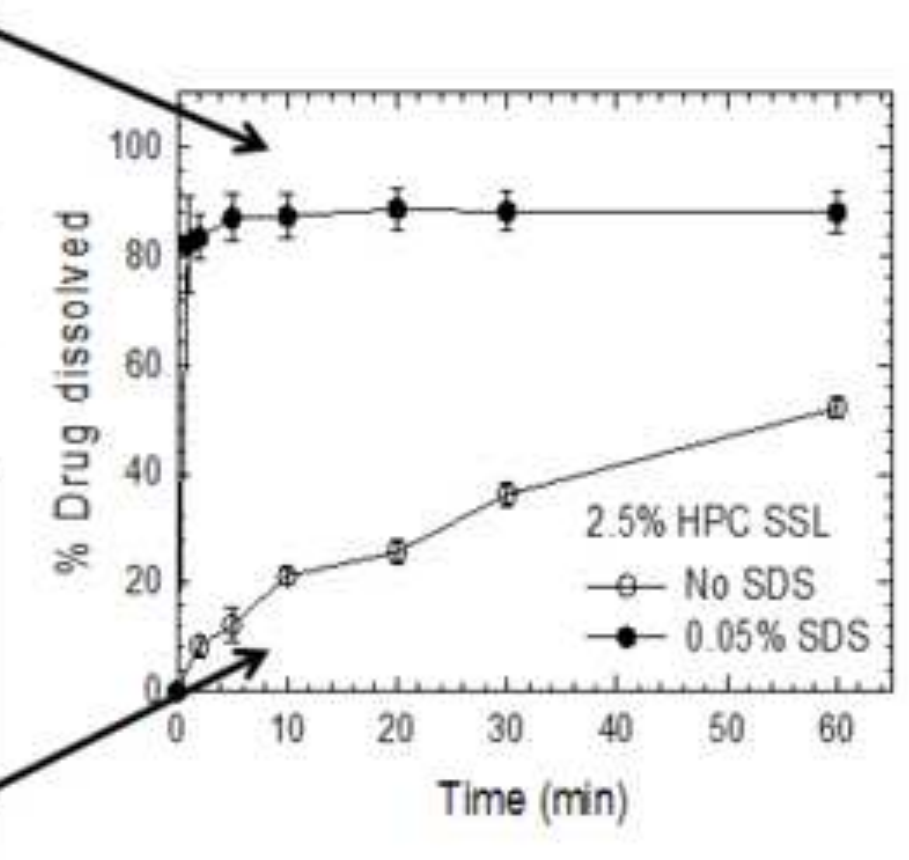

Dissolution profiles of drug composites

$r_{2} 2$ Polymer, hydroxypropyl cellulose $(\mathrm{HPC})$

a Anionic surfactant, sodium dodecyl sulfate (SDS)

- Drug particle, griseofulvin (GF) 